\title{
A meshfree thin shell method for nonlinear dynamic fracture
}

\author{
T.Rabczuk ${ }^{\dagger}$, P.M.A.Areias ${ }^{+}$, T.Belytschko ${ }^{*}, \|$ \\ Department of Mechanical Engineering, Northwestern University, Evanston, IL 60208-311, U.S.A
}

\begin{abstract}
SUMMARY
A meshfree method for thin shells with finite strains and arbitrary evolving cracks is described. The $C^{1}$ displacement continuity requirement is met by the approximation, so no special treatments for fulfilling the Kirchhoff condition are necessary. Membrane locking is eliminated by the use of a cubic or quartic polynomial basis. The shell is tested for several elastic and elasto-plastic examples and shows good results. The shell is subsequently extended to modelling cracks. Since no discretization of the director field is needed, the incorporation of discontinuities is easy to implement and straight forward. Copyright (c) 2000 John Wiley \& Sons, Ltd.
\end{abstract}

KEY WORDS: meshfree methods, cracks, cohesive models, KL constraint, shell

\section{INTRODUCTION}

This paper describes a meshfree method for treating the dynamic fracture of shells. It includes both geometric and material nonlinearities and also includes a meshfree fluid model, so that complex fluid-structure interaction problems are feasible. Here we illustrate this capability with the fracture of a fluid-filled cylinder that is impacted by a penetrating projectile. The shell formulation is based on the Kirchhoff-Love (KL) theory.

A meshfree thin shell based on the imposition of the KL constraints was first proposed by Krysl and Belytschko [1]. However, the shell was developed for small deformations, small strains and elasticity. Three dimensional modelling of shear deformable shells and degenerated shells in a meshfree context was studied by Noguchi et al. [2], Li et al. [3] and Kim et al. [4]. Usually, a low order polynomial basis was used, e.g. in [3], a trilinear polynomial basis was applied and the method was applied to several non-linear problems. However, for thin structures, three dimensional modelling of shell structures is computationally expensive. Garcia et al. [5] developed meshfree methods for plates and beams; the higher continuity of meshfree shape functions was exploited for Mindlin-Reisner plates in combination with a p-enrichment. Wang and Chen [6] proposed a meshfree method for Mindlin-Reisner plates. Locking is treated using

${ }^{*}$ Correspondence to: T. Belytschko, Department of Mechanical Engineering, Northwestern University, 2145 Sheridan Road, Evanston, IL 60208-311, U.S.A. E-mail: t-belytschko@nwu.edu

|| Walter P.Murphy, Professor of Computational Mechanics

$\dagger,+$ Post-Doctoral Research Fellow, Department of Mechanical Engineering 
second order polynomials for the approximation of the translational and rotational motion in combination with a curvature smoothing stabilization. Kanok-Nukulchai et al. [7] addressed shear locking for plates and beams in the element-free Galerkin method.

We develop a meshfree thin shell that combines classical shell theory with a continuum based shell. The kinematic assumptions of classical KL shell theory is adopted. We make use of the generality provided by the continuum description, so that constitutive models developed for continua are easily applicable to shells. The formulation is valid for finite strains.

We include in the shell the capability to model cracks, which are modeled either by cracked particles as in Rabczuk and Belytschko [8] or by a local partition of unity [9, 10, 11]. Due to the higher order continuity of meshfree methods, which enables the use of KirchhoffLove shell theories in pristine form, the incorporation of discontinuities is very simple and straight forward. The director field is not discretized, which simplifies the incorporation of discontinuities. In our meshfree model, the concepts for modelling cracks in continua can be adopted directly to shells.

The paper is arranged as follows: First, the kinematics of the shell is described. Then, the meshfree method, the element-free Galerkin (EFG) method, is reviewed and the concept how to incorporate continuum constitutive models is described. The extensions to modelling cracks is described in section 5. Finally, we test the meshfree shell for different elastic linear and nonlinear problems, plastic problems and cracking problems.

\section{SHELL MODEL}

\subsection{Kinematics}

Consider a body $\Omega$ with material points $\mathbf{X} \in \Omega_{0}$ of the shell in the reference configuration with discontinuities, e.g. cracks, on lines $\Gamma_{0}^{c}$. The boundary is denoted by $\Gamma_{0}$, where $\Gamma_{0}^{u}$ and $\Gamma_{0}^{t}$ are the complementary boundaries on which, respectively, displacements and tractions are prescribed. We consider a surface parametrized by two independent variables $\theta^{\alpha}, \alpha=1$ to 2 ; the surface in the reference (initial) configuration is described by $\mathbf{R}\left(\theta^{\alpha}\right), \mathbf{R} \in \Re^{3}$. The material points in the reference configuration are given by

$$
\mathbf{X}\left(\theta^{i}\right)=\mathbf{R}\left(\theta^{\alpha}\right)+\theta^{3} \frac{d}{2} \mathbf{N}\left(\theta^{\alpha}\right)
$$

where $\theta^{i}$ are curvilinear coordinates, $-1 \leq \theta^{3} \leq 1, d$ is the thickness of the shell, $\mathbf{N}$ is the shell normal and $\mathbf{R}$ is a point on the mid-surface of the shell in the reference configuration $\mathcal{S}_{0}$. Upper Latin and Greek indices range from 1 to 3 and from 1 to 2, respectively and refer to quantities in the cartesian or curvilinear coordinate system. The current configuration is given by

$$
\mathbf{x}\left(\theta^{i}, t\right)=\mathbf{r}\left(\theta^{\alpha}, t\right)+\theta^{3} \frac{d}{2} \mathbf{n}\left(\theta^{\alpha}, t\right)
$$

where $t$ is the time, $\mathbf{n}$ is the director field and $\mathbf{r}$ is a point on the mid-surface position in the current configuration. The first and second fundamental forms are given by

$$
\begin{gathered}
A_{\alpha \beta}=\mathbf{R}_{, \alpha} \cdot \mathbf{R}_{, \beta} \\
B_{\alpha \beta}=\mathbf{R}_{, \alpha, \beta} \cdot \mathbf{N}=-\mathbf{R}_{, \alpha} \cdot \mathbf{N}_{, \beta}
\end{gathered}
$$


The curvilinear coordinates $\theta^{\alpha}$ are such that $\mathbf{R}_{, \alpha}$ form a basis for the tangent space in $\mathbf{X} \in \mathcal{S}_{0}$. For arbitrary $\theta^{3}$, we define a family of surfaces $\mathcal{S}\left(\theta^{3}\right)$ with $\mathcal{S}_{0}=\mathcal{S}(0)$ for which the tangent basis is established from (1) as $\boldsymbol{X}_{, \alpha}=\boldsymbol{R}_{, \alpha}+\frac{\theta^{3}}{2} \boldsymbol{N}_{\alpha}$. We extend this basis by including $\boldsymbol{N}=\boldsymbol{X}_{, 3} \frac{2}{d}$. The resulting basis spans $\Omega_{0}$, and we can then define the metric of $\Omega_{0}$ as $G_{i j}=\boldsymbol{X}_{, i} \cdot \boldsymbol{X}_{, j}$. The dual basis is given by $\boldsymbol{G}^{i}=G^{i j} \boldsymbol{X}_{, j}$ with $\left[G^{i j}\right]=\left[G_{i j}\right]^{-1}$.

The Cauchy-Green tensor is

$$
\boldsymbol{C}=\boldsymbol{F}^{T} \boldsymbol{F}=\underbrace{\left(\boldsymbol{x}_{, i} \cdot \boldsymbol{x}_{, j}\right)}_{C_{i j}} \boldsymbol{G}^{i} \otimes \boldsymbol{G}^{j}
$$

where $\mathbf{x}$ are the spatial position coordinates and $\boldsymbol{F}$ is the deformation gradient.

The Kirchhoff-Love hypothesis is imposed by requiring that $\mathbf{n}$ is perpendicular to $\mathbf{r}_{, \alpha}$, $\alpha=1,2$ :

$$
\mathbf{n}=\frac{\mathbf{r}_{, 1} \times \mathbf{r}_{, 2}}{\left\|\mathbf{r}_{, 1} \times \mathbf{r}_{, 2}\right\|}
$$

\subsection{Virtual work}

The weak form of the momentum equation is written with the principle of virtual work (see e.g. Belytschko et al. [12]): find $\mathbf{r} \in \mathcal{V}$ such that

$$
\delta W=\delta W_{i n t}-\delta W_{e x t}+\delta W_{k i n}-\delta W_{E}=0 \quad \forall \delta \mathbf{r} \in \mathcal{V}_{0}
$$

where

$$
\begin{aligned}
& \mathcal{V}=\left\{\mathbf{r}(\cdot, t) \mid \mathbf{r}(\cdot, t) \in \mathcal{H}^{2}\left(\Omega_{0} / \Gamma_{0}^{c}\right), \quad \mathbf{r}(\cdot, t)=\overline{\mathbf{r}}(t) \text { on } \Gamma_{0}^{u}, \mathbf{r} \text { discontinuous on } \Gamma_{0}^{c}\right\} \\
& \mathcal{V}_{0}=\left\{\delta \mathbf{r} \mid \delta \mathbf{r} \in \mathcal{V}, \delta \mathbf{r}=0 \quad \text { on } \quad \Gamma_{0}^{u}, \quad \delta \mathbf{r} \text { discontinuous on } \Gamma_{0}^{c}\right\} \\
& \delta W_{i n t}=\int_{\Omega_{0} \backslash \Gamma_{0}^{c}}\left\{s^{\alpha \beta} \mathbf{x}_{, \alpha} \cdot \delta \mathbf{x}_{, \beta}\left[\mathbf{G}_{1} \cdot\left(\mathbf{G}_{2} \times \mathbf{G}_{3}\right)\right]\right\} d \Omega \\
& \delta W_{e x t}=\int_{\Omega_{0} \backslash \Gamma_{0}^{c}} \varrho_{0} \mathbf{b} \cdot \delta \mathbf{u} d \Omega_{0}+\int_{\Gamma_{0}^{t}} \overline{\mathbf{t}}_{0} \cdot \delta \mathbf{u} d \Gamma_{0} \\
& \delta W_{E}=\int_{\Gamma_{c}} \overline{\mathbf{t}}_{c} \cdot \delta[[\mathbf{u}]] d \Gamma \\
& \delta W_{k i n}=\int_{\Omega_{0} \backslash \Gamma_{0}^{c}} \varrho_{0} \delta \mathbf{u} \cdot \ddot{\mathbf{u}} d \Omega_{0}
\end{aligned}
$$

where the prefix $\delta$ identifies the test function and $W_{\text {ext }}$ is the external energy, $W_{\text {int }}$ designates the internal energy, $W_{E}$ is the crack cohesive energy and $W_{k i n}$ the kinetic energy, $\varrho_{0}$ is the density, $\mathbf{s}$ is the Kirchhoff stress, $\mathbf{b}$ is the body force and $\overline{\mathbf{t}}_{0}$ the prescribed traction; superposed dots denote material time derivatives.

For details of the discrete equations see Rabczuk and Belytschko [8, 13, 14], Belytschko et al. [15]. 


\subsection{Discretization}

The approximation of the shell surface is given by

$$
\mathbf{r}\left(\theta^{\alpha}, t\right)=\sum_{I \in \mathcal{W}} \Phi_{I}\left(\theta^{\alpha}\right) \mathbf{r}_{I}(t)
$$

where $\Phi_{I}\left(\theta^{\alpha}\right)$ are the shape functions and $\mathcal{W}$ is the domain of influence of the corresponding particle.

We require $C^{3}$ displacement continuity in the meshfree method. So there is no need to discretize the director field $\mathbf{n}$ and $\mathbf{n}$ is readily obtained from eq. (6). The variation of the motion $\mathbf{x}$ (and their spatial derivatives) is given by

$$
\delta \mathbf{x}=\delta \mathbf{r}+\theta^{3} \frac{d}{2} \delta \mathbf{n}
$$

The variation of the normal can be expressed in terms of $\mathbf{r}$ :

$$
\delta \mathbf{n}=a_{1} \boldsymbol{a}_{4} \cdot \boldsymbol{a}_{2}
$$

where $a_{1}=\frac{1}{\left\|\boldsymbol{r}_{, 1} \times \boldsymbol{r}_{, 2}\right\|}, \boldsymbol{a}_{2}=\boldsymbol{r}_{, 1} \times \delta \boldsymbol{r}_{, 2}-\boldsymbol{r}_{, 2} \times \delta \boldsymbol{r}_{, 1}$ and $\boldsymbol{a}_{4}=(\boldsymbol{I}-\boldsymbol{n} \otimes \boldsymbol{n})$.

The derivatives of the motion $\mathbf{x}$ are given by

$$
\mathbf{x}_{, \alpha}=\underbrace{\sum_{I \in \mathcal{W}} \Phi_{I, \alpha} \mathbf{r}_{I}}_{\mathbf{r}_{, \alpha}}+\theta^{3} \frac{d}{2} \mathbf{n}_{, \alpha}
$$

with

$$
\mathbf{n}_{, \gamma}=a_{1} \boldsymbol{a}_{4} \cdot \boldsymbol{a}_{5}
$$

with $\boldsymbol{a}_{5}=\boldsymbol{r}_{, 1} \times \boldsymbol{r}_{, 2 \gamma}-\boldsymbol{r}_{, 2} \times \boldsymbol{r}_{, 1 \gamma}$.

To obtain the variation $\delta \mathbf{x}_{, \alpha}$, the derivatives of the variation of the normal have to be computed. Considering eq. (15), the derivatives are:

$$
\begin{gathered}
\delta \mathbf{n}_{, \gamma}=-a_{1}^{3}\left[\left(\boldsymbol{r}_{, 1} \times \boldsymbol{r}_{, 2}\right) \cdot \boldsymbol{a}_{5}\right]\left(\boldsymbol{a}_{4} \cdot \boldsymbol{a}_{2}\right)-2 a_{1}\left(\boldsymbol{n} \otimes \boldsymbol{a}_{5}\right)^{\mathrm{S}} \cdot \boldsymbol{a}_{2}+ \\
a_{1} \boldsymbol{a}_{4} \cdot \delta \boldsymbol{a}_{5}
\end{gathered}
$$

\section{EFG-MESHFREE METHOD}

We use the element free Galerkin (EFG) method that is based on a moving least square (MLS) approximation, see e.g. Belytschko et al. [16], Belytschko and Lu [17]. For the meshfree shell, at least a quadratic polynomial basis is required. Krysl and Belytschko [1] showed that a quadratic basis can lead to membrane locking in meshfree thin shells and showed that a quartic basis completely eliminates membrane locking. A quartic polynomial basis can be written as:

$$
\begin{array}{r}
\mathbf{p}\left(\theta^{\alpha}\right)=\left(1, \theta^{1}, \theta^{2},\left(\theta^{1}\right)^{2}, \theta^{1} \theta^{2},\left(\theta^{2}\right)^{2},\left(\theta^{1}\right)^{3},\left(\theta^{1}\right)^{2} \theta^{2},\left(\theta^{2}\right)^{2} \theta^{1}, \ldots\right. \\
\left.\ldots\left(\theta^{2}\right)^{3},\left(\theta^{1}\right)^{4},\left(\theta^{1}\right)^{3} \theta^{2},\left(\theta^{1}\right)^{2}\left(\theta^{2}\right)^{2}, \theta^{1}\left(\theta^{2}\right)^{3},\left(\theta^{2}\right)^{4}\right)
\end{array}
$$


For our examples, it was sufficient to use a cubic basis.

The MLS approximation is given by:

$$
r_{i}^{h}\left(\theta^{\alpha}, t\right)=\sum_{J \in S} \mathbf{p}\left(\theta_{J}^{\alpha}\right) \mathbf{a}_{i}\left(\theta^{\alpha}, t\right)
$$

with $\mathbf{a}_{i}\left(\theta^{\alpha}, t\right)$ chosen to minimize

$$
\mathcal{J}_{i}=\sum_{J \in S}\left(\mathbf{p}\left(\theta_{J}^{\alpha}\right)^{T} \mathbf{a}_{i}\left(\theta^{\alpha}, t\right)-r_{J i}(t)\right)^{2} w\left(\theta^{\alpha}-\theta_{J}^{\alpha}, h\right) .
$$

with respect to $\mathbf{a}_{i}\left(\theta^{\alpha}, t\right)$. This leads to the approximation

$$
r_{i}^{h}\left(\theta^{\alpha}, t\right)=\sum_{J \in S} r_{J i}(t) \Phi_{J}\left(\theta^{\alpha}\right)
$$

with

$$
\begin{gathered}
\Phi_{J}=\mathbf{p}^{T}\left(\theta^{\alpha}\right) \cdot \mathbf{M}^{-1}\left(\theta^{\alpha}\right) \cdot \mathbf{p}\left(\theta_{J}^{\alpha}\right) w\left(\theta^{\alpha}-\theta_{J}^{\alpha}, h\right) \\
\mathbf{M}\left(\theta^{\alpha}\right)=\sum_{J \in S} \mathbf{p}\left(\theta_{J}^{\alpha}\right) \mathbf{p}^{T}\left(\theta_{J}^{\alpha}\right) w\left(\theta^{\alpha}-\theta_{J}^{\alpha}, h\right) .
\end{gathered}
$$

$w(r)=w\left(\theta_{J}^{\alpha}-\theta_{I}^{\alpha}, h\right)$ is the kernel function that determines the order of continuity. In the EFG method, the continuity of the shape functions is equivalent to the continuity of the kernel, see [18]. A cubic spline kernel leads to $C^{2}$ continuity, a quartic spline to $C^{3}$ continuity. Recall that for a Kirchhoff shell, $C^{1}$ continuity is required.

For rectangular plates and for cylinders, the Jacobian is constant and hence $\theta^{i}$ are linear combinations of $\mathbf{x}$ and linear completeness is guaranteed. It is noted that the same shape functions are employed in shape and displacement approximations to guarantee strain-free states in rigid body motion, see Krysl and Belytschko [1].

\section{CONTINUUM CONSTITUTIVE MODELS}

For the constitutive model, we adopt the algorithm of table I. We use a two-dimensional nonsymmetric radial return and rotate so that the 3-3 component corresponds to the normal. The normal strain (and consequently the normal stress) is filtered, according to figure 1. For more details on that specific constitutive model, the reader is referred to Areias et al. [19].

\section{CRACK MODELING}

The approach is extended to shells with cracks by enriching the mid-surface motion $\mathbf{r}\left(\theta^{\alpha}, t\right)$ with a discontinuous function. The jump in the director field is then obtained directly via the discontinuous part of $\mathbf{r}\left(\theta^{\alpha}, t\right)$. The fact that there is no need to discretize the director field, facilitates the incorporation of discontinuities in shells.

The force introduced here corresponds to the resistance to opening, which is a function of the opening displacement. The opening displacement can be written as a function of the mid-surface position and the director. 


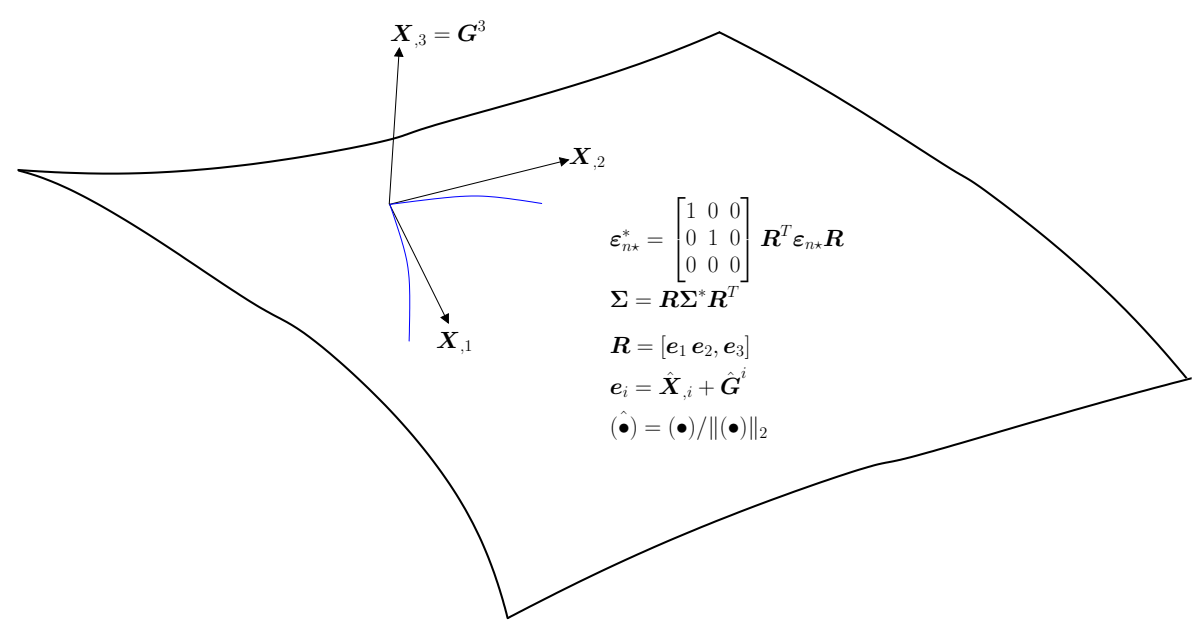

Figure 1. Return mapping: filtering of the normal strain and stress.

We use two methods; both were already used to model cracks in continua, see Rabczuk and Belytschko [8], Ventura et al. [11], Rabczuk and Belytschko [13]. The last one was used in linear fracture mechanics and is here modified to deal with cohesive cracks.

\subsection{Method 1: Cracked particles}

To model cracks, the approximation for $\mathbf{r}$ is enriched with a discontinuous function, so

$$
\mathbf{r}\left(\theta^{\alpha}, t\right)=\mathbf{r}^{\mathrm{cont}}\left(\theta^{\alpha}, t\right)+\mathbf{r}^{\mathrm{enr}}\left(\theta^{\alpha}, t\right)
$$

where $\mathbf{r}^{\text {cont }}\left(\theta^{\alpha}, t\right)$ is given by eq. $(22)$. Let $\mathcal{N}$ be the total set of paticles in the discretization and $\mathcal{N}_{c}$ the set of cracked particles. The set of cracked particles consists of the particles where a fracture nucleation or propagation criterion has been met. To model the discontinuous part of the displacement, the test and trial functions are enriched with sign functions which are parametrized by $\delta \mathbf{q}_{I}$ and $\mathbf{q}_{I}$, respectively. The crack surface is assumed to be normal to the reference surface $\mathcal{S}_{0}$. Only cracked particles are enriched, so that the trial and test functions are:

$$
\begin{aligned}
\mathbf{r}\left(\theta^{\alpha}, t\right) & =\sum_{I \in \mathcal{N}} \Phi_{I}\left(\theta^{\alpha}\right) \mathbf{r}_{I}(t)+\sum_{I \in \mathcal{N}_{c}} \Phi_{I}\left(\theta^{\alpha}\right) H\left(f_{I}\left(\theta^{\alpha}\right)\right) \mathbf{q}_{I}(t) \\
\delta \mathbf{r}\left(\theta^{\alpha}\right) & =\sum_{I \in \mathcal{N}} \Phi_{I}\left(\theta^{\alpha}\right) \delta \mathbf{r}_{I}+\sum_{I \in \mathcal{N}_{c}} \Phi_{I}\left(\theta^{\alpha}\right) H\left(f_{I}\left(\theta^{\alpha}\right)\right) \delta \mathbf{q}_{I}
\end{aligned}
$$

where $\mathcal{N}$ is the total set of particles, $\mathcal{N}_{c}$ is the set of cracked particles and $f_{I}\left(\theta^{\alpha}\right)$ is given by

$$
f_{I}\left(\theta^{\alpha}\right)=\mathbf{m} \cdot\left(\theta^{\alpha}-\theta_{I}^{\alpha}\right)
$$

where $\mathbf{m}$ is the normal to the crack. The sign function $H\left(f\left(\theta^{\alpha}\right)\right)$ is defined as:

$$
H\left(f_{I}\left(\theta^{\alpha}\right)\right)=\left\{\begin{array}{cc}
1 & \forall f_{I}\left(\theta^{\alpha}\right)>1 \\
-1 & \forall f_{I}\left(\theta^{\alpha}\right)<1
\end{array}\right.
$$


Table I. The return mapping in the material setting; encapsulation of the small strain case

Make $\boldsymbol{C}_{0}^{p}=\boldsymbol{I}$ (and therefore $\boldsymbol{C}_{0}^{p^{-1}}=\boldsymbol{I}$ ) and $\boldsymbol{\xi}_{0}=\mathbf{0}$ for all quadrature points For each quadrature point at time-step $n$, perform the following calculations 1) Using the current position field $\boldsymbol{x}$ calculate $\boldsymbol{C}_{n+1}=\left(\boldsymbol{x}_{, \alpha} \cdot \boldsymbol{x}_{, \beta}\right) \boldsymbol{G}^{\alpha} \otimes \boldsymbol{G}^{\beta}$ with $\boldsymbol{G}^{\alpha}=G^{\alpha \beta} \boldsymbol{X}_{, \beta}$ and $\left[G^{\alpha \beta}\right]=\left[\boldsymbol{X}_{, \alpha} \cdot \boldsymbol{X}_{, \beta}\right]^{-1}$

2) Calculate the trial of the elastic measure $\boldsymbol{C}_{n \star}^{E}=\boldsymbol{C}_{n+1} \boldsymbol{C}_{n}^{p^{-1}}$

3) Use a second order Padé approximation to calculate $\varepsilon_{n \star}=\frac{1}{2} \ln C_{n \star}^{E}$

4) Using a modified (unsymmetric) small strain return-mapping algorithm, update $\boldsymbol{\xi}_{n}$ and calculate $\boldsymbol{\varepsilon}_{n+1} \Delta \alpha, \boldsymbol{\Sigma}$ and the small strain consistent modulus $\mathbb{C}$

5) Calculate the new plastic metric inverse as $\boldsymbol{C}_{n+1}^{p-1}=\boldsymbol{C}_{n+1}^{-1} \exp \left[2 \boldsymbol{\varepsilon}_{n+1}\right]$ using a first order Padé approximation for the exponential function

6) Calculate the contravariant components of the stress as

$$
s^{\alpha \beta}=\boldsymbol{G}^{\alpha} \cdot\left(\boldsymbol{C}^{-1} \boldsymbol{\Sigma} \boldsymbol{G}^{\beta}\right)
$$

and the contravariant components of the tangent modulus as

$$
C^{\alpha \beta \gamma \delta}=G^{\alpha i} G^{\beta j} G^{\gamma k} G^{\delta l} \mathfrak{C}_{i j k l}
$$

where $\mathfrak{C}_{i j k l}$ is the elasticity tensor and $G^{\alpha i}=\boldsymbol{G}^{\alpha} \cdot \boldsymbol{e}_{i}$ for any $i=1,2,3$ and $\alpha=1,2 . \boldsymbol{\xi}_{0}$ are a set of internal variables, $\alpha$ is the plastic parameter of the yield surface and $\boldsymbol{\Sigma}$ are the elastic stresses.

Note that, in general, different shape functions can be used for the continuous and discontinuous parts. Since at least second order complete basis polynomials have to be used, the domains of influence are large and the cracked particles will influence more particles than in the continuum version of this method [8]. Note also, that in [8, 13], the method was developed for a stress point integration where stresses are evaluated at nodes and stress points. In this approach, the nodal stresses are obtained by MLS fits.

\subsection{Method 2: Local Partition of Unity approach}

In this approach, we enrich the test and trial functions with additional unknowns so that the crack is continuous and includes branch functions at the tip as in Moes et al. [10], Ventura et al. [11]. Therefore, the test and trial functions in terms of a signed distance function $f$, see figure 2, are 


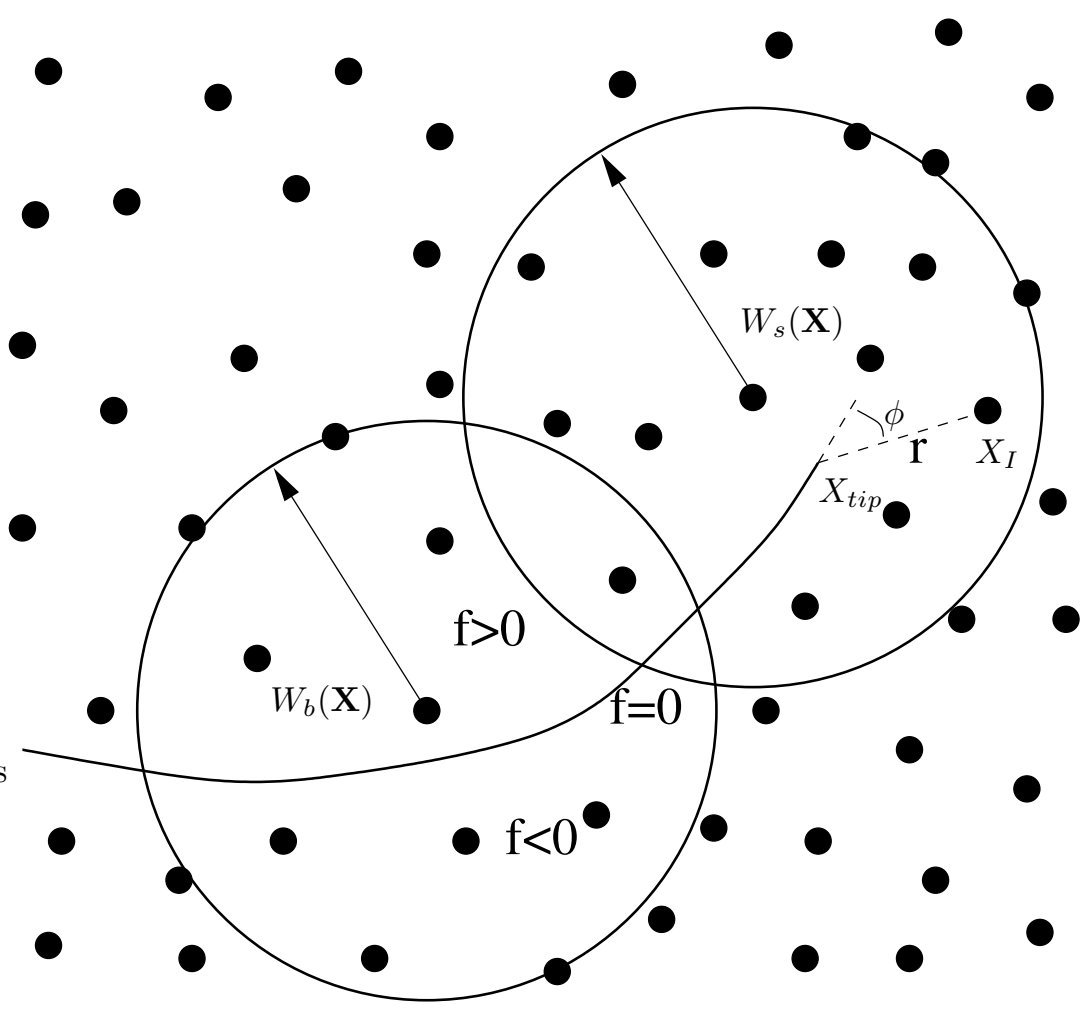

Figure 2. Crack with partially cut and complete cut domain of influence particles

$$
\begin{aligned}
\mathbf{r}\left(\theta^{\alpha}, t\right) & =\sum_{I \in W\left(\theta^{\alpha}\right)} \Phi_{I}\left(\theta^{\alpha}\right) \mathbf{r}_{I}(t)+\sum_{I \in W_{b}\left(\theta^{\alpha}\right)} \Phi_{I}\left(\theta^{\alpha}\right) H\left(f_{I}\left(\theta^{\alpha}\right)\right) \mathbf{q}_{I}(t) \\
& +\sum_{I \in W_{s}\left(\theta^{\alpha}\right)} \Phi_{I}\left(\theta^{\alpha}\right) \sum_{K} \mathbf{B}_{K}\left(\theta^{\alpha}\right) \mathbf{b}_{K I}(t) \\
\delta \mathbf{r}\left(\theta^{\alpha}\right) & =\sum_{I \in W\left(\theta^{\alpha}\right)} \Phi_{I}\left(\theta^{\alpha}\right) \delta \mathbf{r}_{I}+\sum_{I \in W_{b}\left(\theta^{\alpha}\right)} \Phi_{I}\left(\theta^{\alpha}\right) H\left(f_{I}\left(\theta^{\alpha}\right)\right) \delta \mathbf{q}_{I} \\
& +\sum_{I \in W_{s}\left(\theta^{\alpha}\right)} \Phi_{I}\left(\theta^{\alpha}\right) \sum_{K} \mathbf{B}_{K}\left(\theta^{\alpha}\right) \delta \mathbf{b}_{K I}
\end{aligned}
$$

The first term on the RHS of equation (30) is the usual approximation where $\Phi_{I}$ are the shape functions, the second and third term is the enrichment. The coefficients $\mathbf{q}_{I}$ and $\mathbf{b}_{I}$ are additional degrees of freedom. Only nodes which are located in the domain $W_{b}\left(\theta^{\alpha}\right)$ are enriched with the additional unknowns. The third term of equations (31) and (30) is applied around the crack tip $W_{s}\left(\theta^{\alpha}\right)$. 


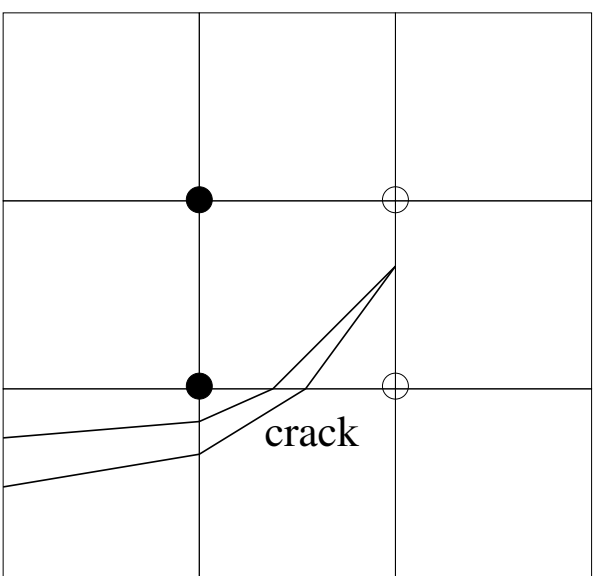

enriched node

un-enriched node

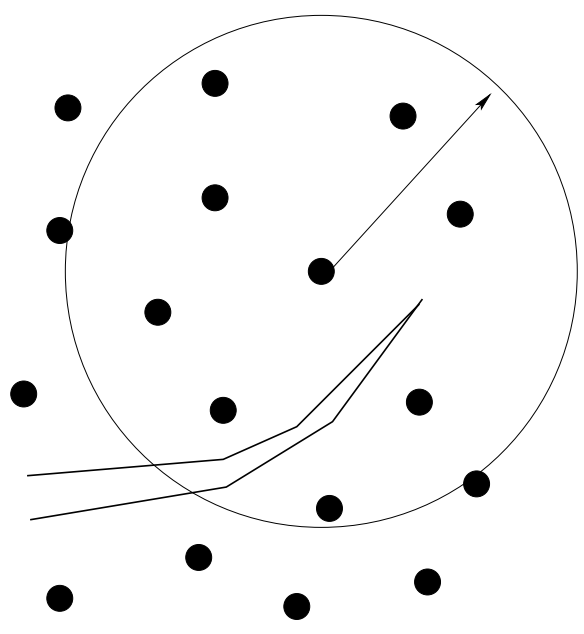

a)

b)

Figure 3. Crack with enriched nodes in XFEM and meshfree methods

For cohesive cracks, the crack tip enrichment is usually omitted and the cohesive forces depend only on the additional unknowns $\mathbf{q}_{I}$. In XFEM, the omission of the third term in eq. (31) is straightforward since it is easily possible to impose the appropriate boundary conditions, see figure 3a. However, in meshfree methods, this technique cannot be applied analogously, see figure $3 \mathrm{~b}$, since there will always be particles with a partially cut domain of influence. Therefore, we introduce the branch function enrichment and use [17]:

$$
\mathbf{B}=\left(r \sin \frac{\phi}{2}\right)
$$

where $r$ is the distance from the crack tip and $\phi$ the angle as shown in figure 2 .

We would like to mention that for particles in the blending region, i.e. the particles whose domain of influence is not cut but that are influenced by the "enriched" particles, only the usual approximation (first term on the RHS of eqs. (31) and (30)), is considered in the approximation of the test and trial functions. For crack propagation, we control the crack length and propagate the crack through an entire background cell.

\subsection{Crack initiation and Cohesive model}

We use the Rankine criterion for crack initiation, i.e. if the principal tensile stress exceeds a given value, we introduce a discontinuity. An alternative is a strain based criterion, meaning if a critical effective plastic strain value is exceeded, a crack is introduced. 


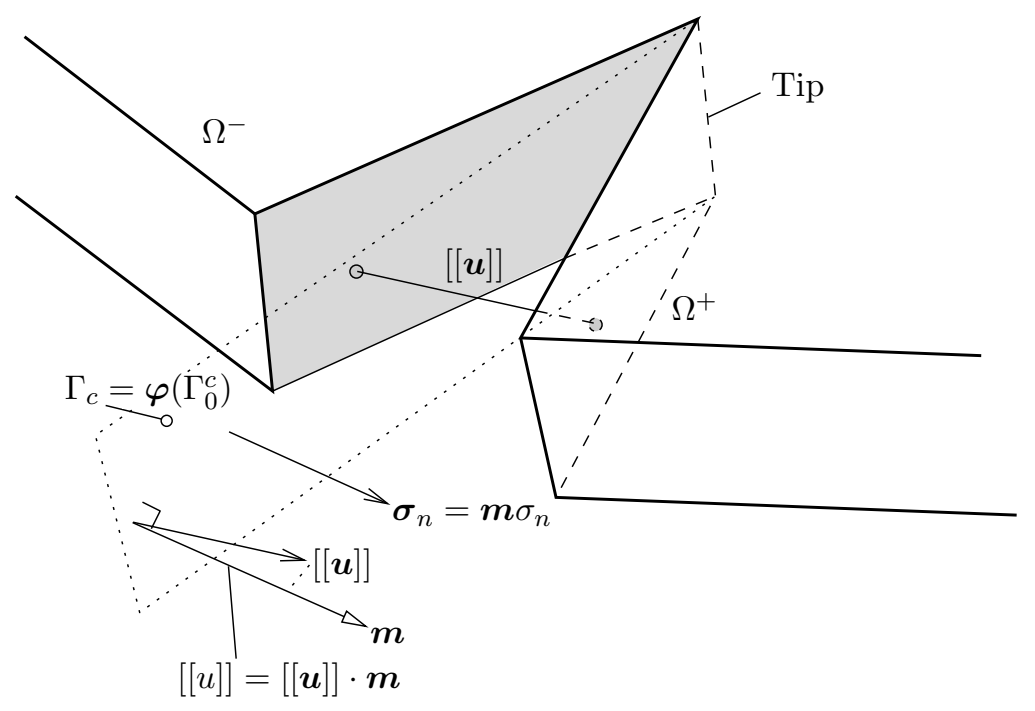

Figure 4. Cohesive forces arising from the crack surface separation.

The virtual work of the cohesive forces is included in the term $\delta W_{E}$. The force introduced by this term corresponds to the resistance to opening, which is a function of the opening displacement itself. Because this type of dissipation mechanism occurs on a set of measure zero, part of the energy dissipated in the continuum is transferred to the cohesive law. The opening displacement can be written as a function of the mid-surface position on both sides of the crack and the director on both sides of the crack. We denote the surface opening by $[[u]]$, which can be expressed as (see figure 4):

$$
[[u]]=[[\boldsymbol{u}]] \cdot \boldsymbol{m}
$$

where $[[\boldsymbol{u}]]$ is the jump in the displacement (discontinuity magnitude) defined by

$$
[[\boldsymbol{u}]]=[[\boldsymbol{r}]]+\theta^{3}[[\boldsymbol{n}]]
$$

with

$$
[[\boldsymbol{n}]]=\frac{\mathbf{r}_{, 1}^{+} \times \mathbf{r}_{, 2}^{+}}{\left\|\mathbf{r}_{, 1}^{+} \times \mathbf{r}_{, 2}^{+}\right\|}-\frac{\mathbf{r}_{, 1}^{-} \times \mathbf{r}_{, 2}^{-}}{\left\|\mathbf{r}_{, 1}^{-} \times \mathbf{r}_{, 2}^{-}\right\|}
$$

where $[[\boldsymbol{r}]]$ is the mid-surface displacement jump, $[[\boldsymbol{n}]]$ is the director field jump and the superimposed + and - denote the two sides of the crack.

If we denote the cohesive virtual work as $\delta W_{E}^{c}$, then we can write it in terms of the Kirchhoff stress $\sigma_{n}$ as:

$$
\delta W_{E}^{c}=-\int_{\Gamma_{O}^{c}} \sigma_{n} \delta[[u]] \mathrm{d} \Gamma=-\int_{\Gamma_{O}^{c}} \boldsymbol{\sigma}_{\boldsymbol{n}} \cdot \delta[[\boldsymbol{u}]] \mathrm{d} \Gamma
$$




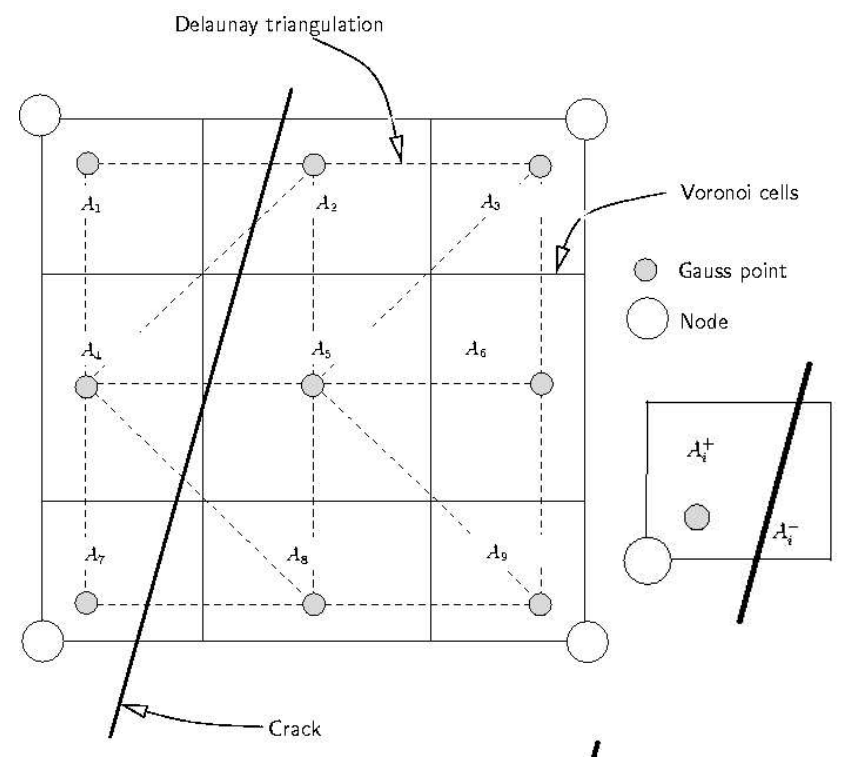

Figure 5. Integration with background cells across the crack

where $\Gamma_{0}^{c}$ here represents the area of the crack surface.

We use the following constitutive model for the cohesive zone [19]:

$$
\sigma_{n}=\frac{\sigma_{\max }}{\epsilon} \exp \left(-\frac{\sigma_{\max }}{G_{f}} \epsilon\right)[[u]]
$$

where $\sigma_{\max }$ is the maximum cohesive stress, $G_{f}$ is the fracture (surface) energy and $\epsilon=$ $\max _{\text {history }}[[u]]$ denotes an internal variable. A penalty term is employed to attenuate crack face inter-penetration.

\subsection{Integration}

We use a background mesh to integrate the governing equations so that a background cell is created by four particles. We use 64 Gauss points in a cell. To integrate the terms across the crack effectively, a sub-triangulation of the background cell through which crack passes is performed. This requires the introduction of sub-triangular integration cells. We will pursue another idea proposed by Song et al. [20] in the context of finite elements and modify the quadrature weights.

Therefore, the element area is subdivided into cells by a Voronoi procedure as shown in figure 5, simplified for the case of 9 Gauss points. This procedure is straight-forward since a Delaunay triangulation is already implemented in the code.

The sum of the area of the Voronoi cells $A_{i}$ will be the area of the background cell $A_{\text {total }}$ in the parent domain that would be 4 in the case of integration in the interval $[-1,1]$ though 
this is not mandatory:

$$
\sum_{i} A_{i}=A_{\text {total }}
$$

The quadrature weights of the Gauss points whose area is not crossed by the discontinuity are unmodified. Otherwise the weights are computed by:

$$
\begin{aligned}
& W_{i}^{+}=W_{i} \frac{A_{i}^{+}}{A_{i}} \\
& W_{i}^{-}=W_{i} \frac{A_{i}^{-}}{A_{i}}
\end{aligned}
$$

\subsection{Essential boundary conditions}

Essential boundary conditions are applied by means of the penalty method or Lagrange multipliers, see e.g. Krysl and Belytschko [1], Huerta et al. [18].

\section{EXAMPLES}

\subsection{Elastic Examples}

6.1.1. Linear example The linear shell problem is shown in figure 6. The shell consists of a pinched cylinder with rigid end diaphragms and has been analyzed, among others, in references [21, 22, 23, 24]; these results are here reproduced for comparison. The point-load displacement is monitored. The results for the 4 node selectively integrated quadrilateral (SRI) with stabilization are taken from reference [25]. A value of $1.82488 \times 10^{-5}$ (c.f. Belytschko et al. [25]) consistent units is adopted as a reference.

The results are shown in figures 7 in terms of the normalized displacements. No membrane locking occurs and the accuracy is comparable with the results of other authors for finer discretizations. Note that a certain number of neighbor particles is necessary, so that we are not able to perform computations with very coarse particle arrangements.

6.1.2. Pinching of a short clamped cylinder Consider a short clamped cylinder similar to the one in figure 6. The cylinder is loaded by two monotonically increasing point loads in opposing directions. This test was carried out e.g. in references [32, 33]. Crisfield and Peng [32] discovered some "artificial" instabilities for coarse meshes in their method. Note that in contrast to e.g. Areias et al. [33] we do not employ any symmetry conditions but model the entire cylinder. The radius is $R=1.016$, thickness $t=0.03$, length $L=3.048$, Poisson ratio $\nu=0.3$ and Young's modulus $E=2.0685 \times 10^{7}$.

We will present the results for two different particle discretizations with 1680 particles and 6560 particles. The load deflection curve for the two different particle discretizations is shown in figure 8 and compared to results of other authors. The results don't show mesh dependence. No instabilities as reported in [32] were observed. Note that no contact is used in this example, i.e. the shell will interpenetrate. This is done in accordance to computations of other authors, see e.g. [32, 33]. 


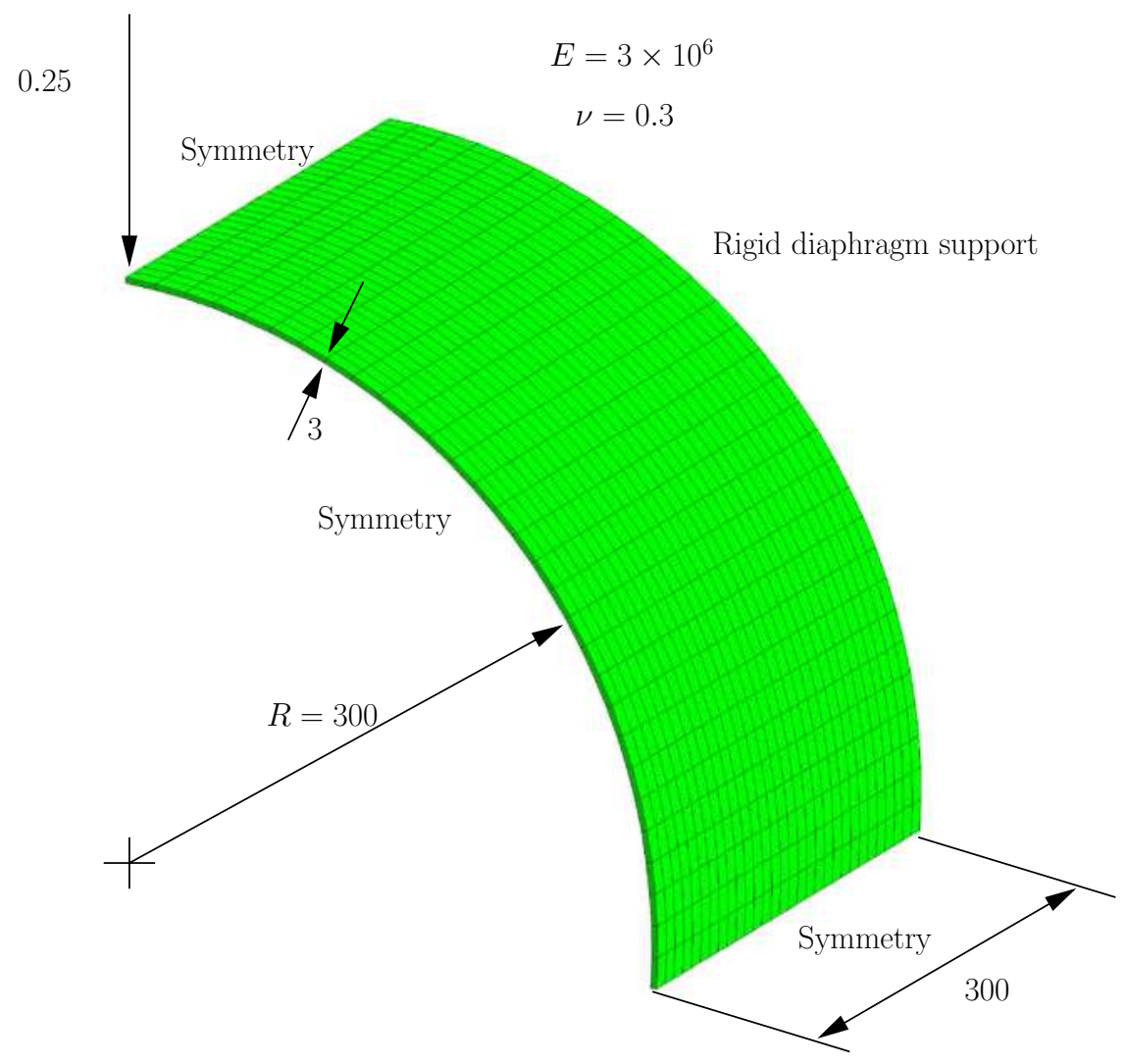

Shell I

Figure 6. Set-up of the linear pinched clamped cylinder

6.1.3. Pullout of an open cylinder This example has been described and studied by various authors (e.g. [36, 37]) and combines bending and membrane effects. It consists of a cylindrical shell with open ends which is pulled at two diametrically opposed points through the application of point loads. The geometry is similar to the one shown in figure 6. The dimensions of the problems are radius $R=4.953$ and length $L=5.175$. In the symmetry plane, a force is applied at point $A$. Note that we do not employ symmetry and discretize the entire cylinder. The material is considered elastic, with Young's modulus $E=10.5 \times 10^{6}$ and Poisson coefficient $\nu=0.3125$, in agreement with the above references. The particle arrangements of the last example are studied, 1680 particles and 6560 particles. The absolute radial displacement for point $\mathrm{A}$ is compared with the values of other authors. Figure 9 shows this comparison. The deformed cylinder for the two discretizations is shown in figure 10. 


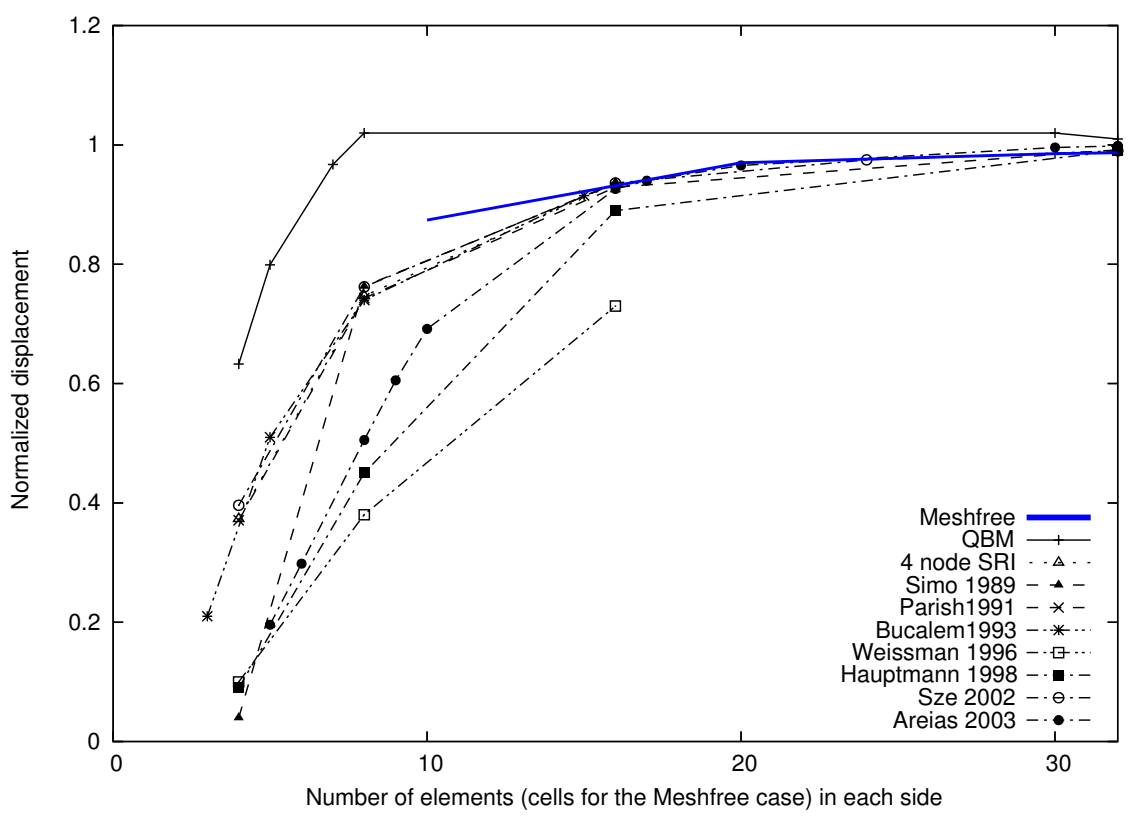

Figure 7. Load-displacement curve of the linear pinched clamped cylinder compared with Areias et al. [26] (QBM), Simo et al. [21], Parish [22], Sze et al. [27], Areias et al. [28], Bucalem and Bathe [29], Hauptmann and Schweizerhof [30], Weissmann [31]

6.1.4. Elastic pinched cylinder with end diaphragms A cylindrical shell with rigid end diaphragms is pinched by two opposite point loads as shown in figure 11. This example was studied by Campello et al. [41]. The material parameters are adopted from [41] and are Young's modulus $E=30.000$ and $\nu=0.3$. The pinching forces are increased in [41] up to $P=12,000$. We increase the pinching forces until self contact. Severe buckling is observed a short time before contact. The load deflection curve is shown in figure 12 and agrees well with results of other authors.

\subsection{Elasto-plastic Examples}

6.2.1. Plastic pinched cylinder with end diaphragms Consider the example from section 6.1.4 again where an elastic cylinder with rigid end diaphragm was loaded by a point load. We study this example with a non-linear material and modify the geometry according to Areias [42]. The total length of the cylinder is 600 with a radius of 300 and a thickness of 3 . We apply the elasto-plastic material described in section 4. The material parameters are Young's modulus $E=3000$ and Poisson ratio $\nu=0.3$. The hardening law is given by $\sigma_{y}=24.3+300 \epsilon_{p}$ where $\sigma_{y}$ is the yield stress and $\epsilon_{p}$ the accumulated effective plastic strain. We adopted the discretization from section 6.1 .4 and use 4,960 particles.

The deformed cylinder with the corresponding effective plastic strain distribution is shown in figure 13. Very large plastic strains occur where the load is applied. The load deflection curve is shown in figure 14 and agrees well with results of other authors. 


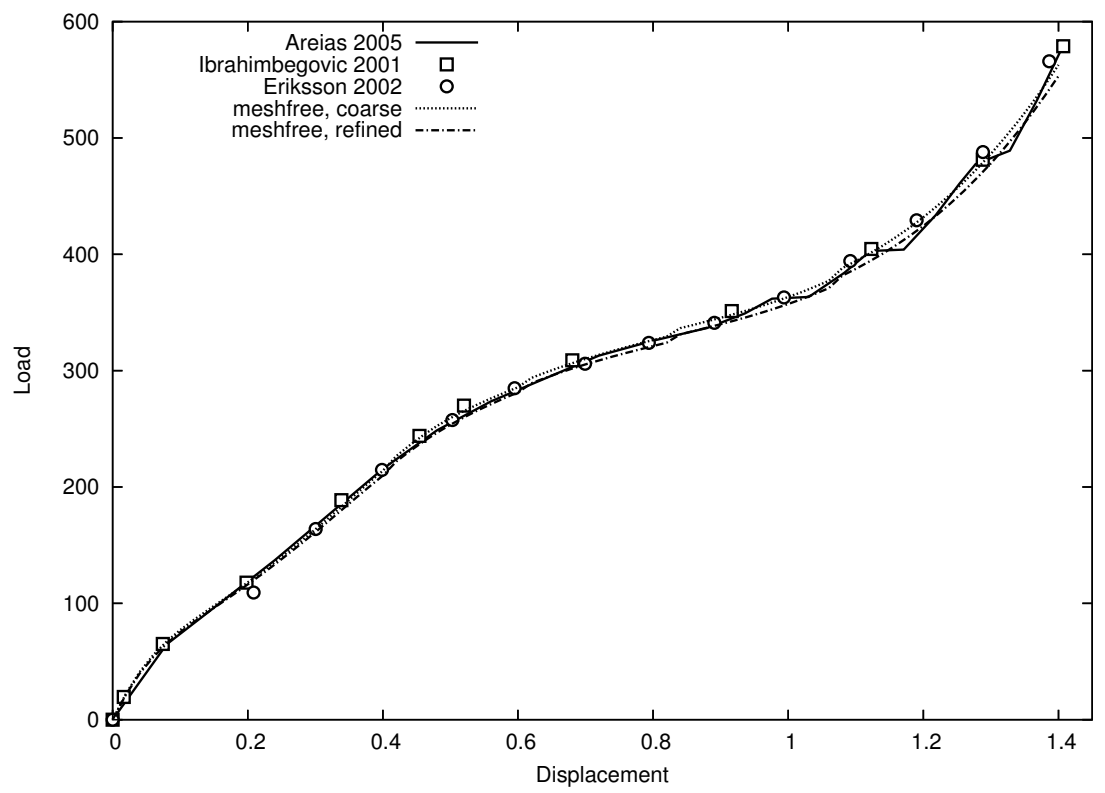

Figure 8. Load-displacement curve of the pinched clamped cylinder compared with Areias et al. [26], Ibrahimbegović et al. [34], Eriksson and Pacoste [35]

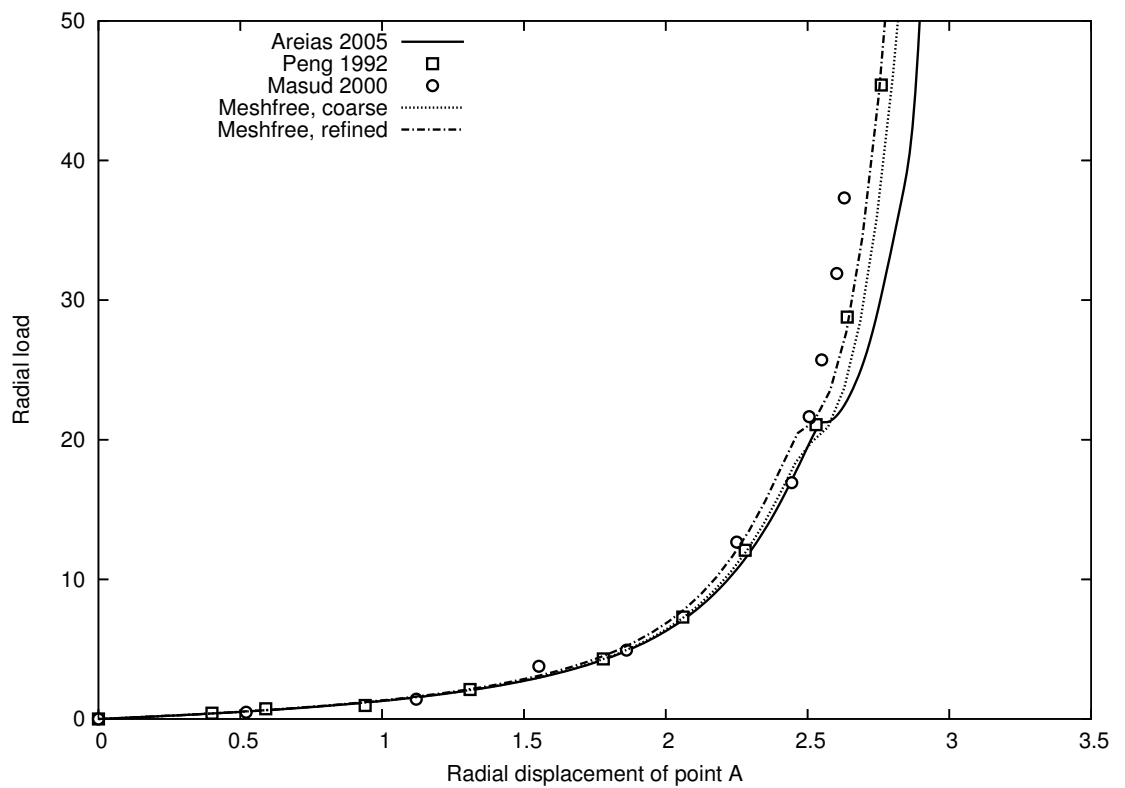

Figure 9. Load-displacement curve of the open pullout cylinder compared with Areias et al. [26], Peng and Crisfield [38], Masud et al. [39] 


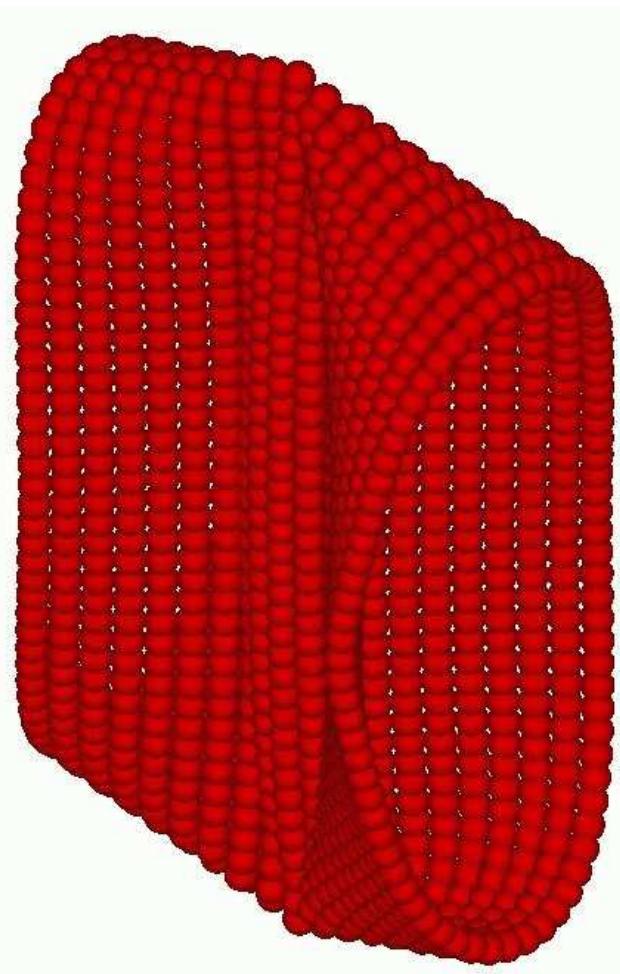

a)

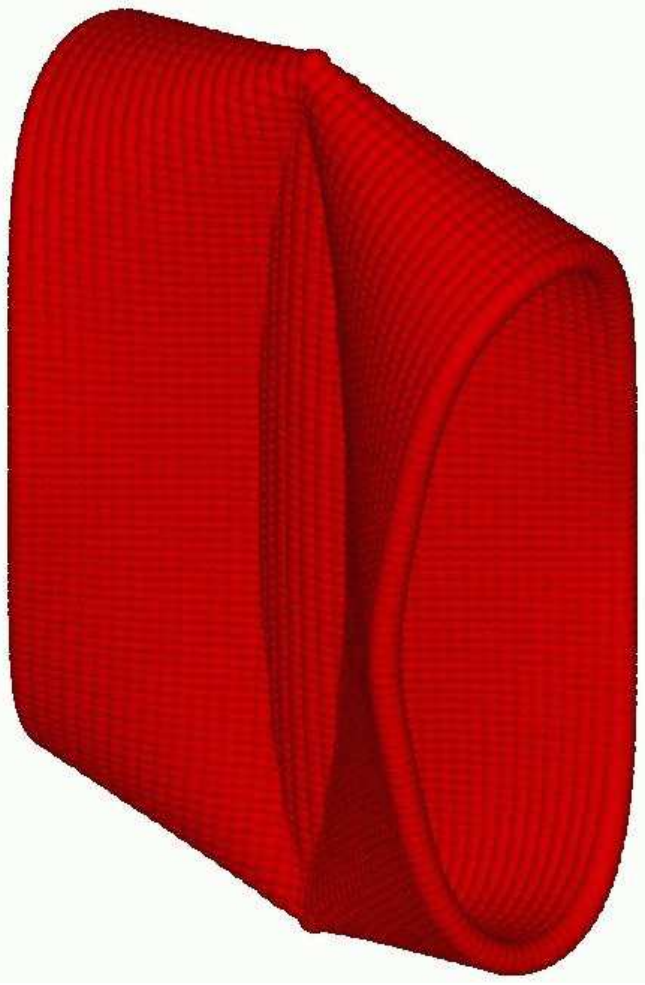

(b)

Figure 10. Deformed open pullout cylinder for different numbers of particles, a) 1680 particles, b) 6560 particles

\subsection{Cracking Examples}

6.3.1. Tearing of a plate by out-of plane loading This example is based on the experiments described in Muscat-Fenech and Atkins [45] and consists of a pre-cracked elastic-plastic plate as shown in figure 15. The yield strength $\sigma_{y}$ is obtained from the hardening law:

$$
\sigma_{y}=a\left(b+\epsilon_{p}\right)^{n}
$$

where $a=574 M P a, b=0.010372$ and $n=0.26$ are measured material parameters and $\epsilon_{p}$ is the effective plastic strain. The constitutive model explained in section 4 is employed.

The test involves large displacements and rotations under the applied forces. We tested four different values of $a_{0}, 30 \mathrm{~mm}, 40 \mathrm{~mm}, 50 \mathrm{~mm}$ and $60 \mathrm{~mm}$. We also tested different refinements, from 6400 particles up to 35,000 particles. We will first show the results for the PU-cracking method. The deformed plate at the end of the computation for 6400 particles is shown in figure 16, for a value of $a_{0}=40 \mathrm{~mm}$ (the other results look similar). The deformed plate shows good agreement with the experimental result. Note that the crack actually passes farther to the end of the plate. Due to the small crack opening, this is not clearly noticeable in figure 16 .

The corresponding load deflection curves are shown in figure $17 \mathrm{a}$. They agree quite well with 


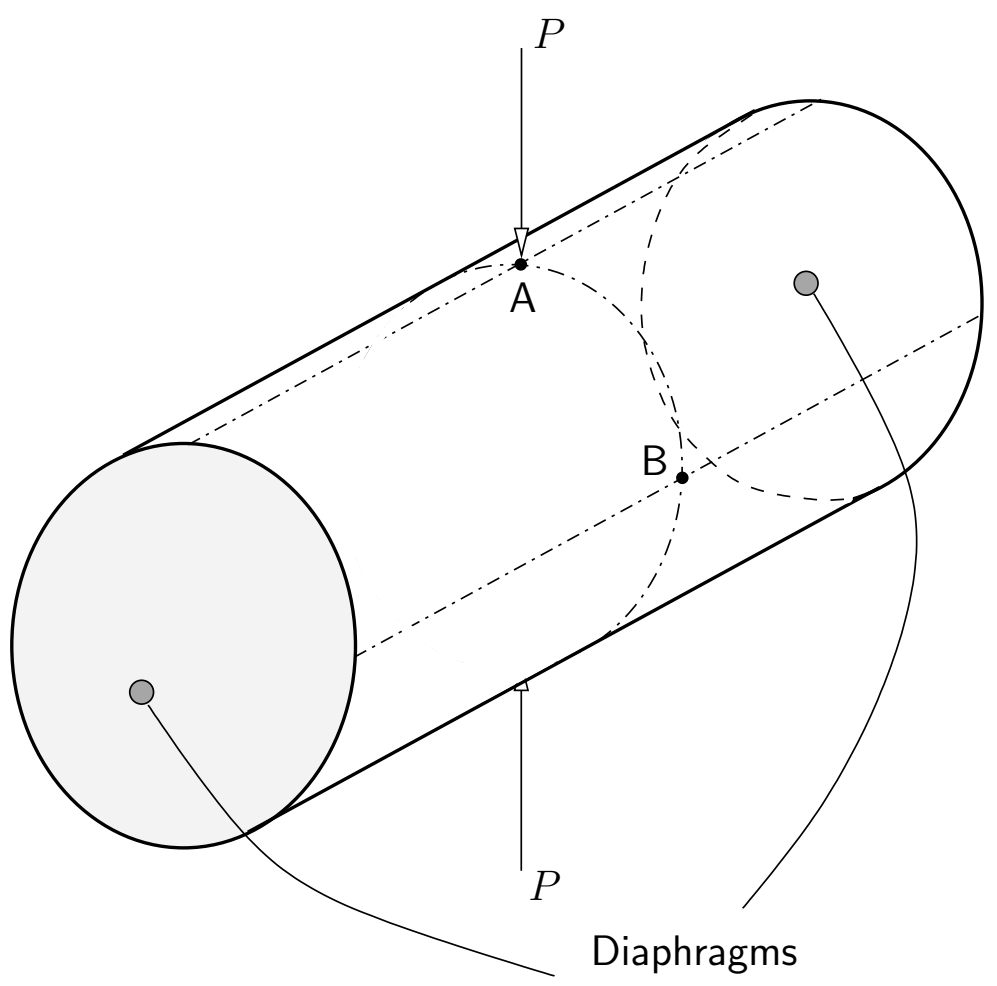

Figure 11. Cylindrical shell with rigid end diaphragm

the experiments. A maximum deflection of approximately $63 \mathrm{~mm}$ is obtained at the end of the simulation. The influence of the refinement is shown in figure $17 \mathrm{~b}$ for values of $a_{0}=40 \mathrm{~mm}$ and $a_{0}=60 \mathrm{~mm}$. No mesh-dependence is observed. The results for the cracked particle method look qualitatively similar but the quantitative agreement is not as good.

6.3.2. Crack path modification at a tear strap in a pressurized cylindrical shell This example consists in the analysis of crack propagation in a pressurized cylinder and is based on the tests carried out by Keesecker et al. [46] and was analysed using enhanced strain/XFEM elements in reference [47]. The cylinder is reinforced with two tear straps (see the above references) whose purpose is to induce "flapping", which consists of crack turning near these tear straps. If the purpose is fulfilled, axially propagating cracks are arrested.

Figure 18 shows the relevant properties. The hardening law is given by the relation $\sigma_{y}=520\left(0.0483+\varepsilon_{p}\right)^{0.0455825}$ where $\sigma_{y}$ is the yield stress and $\varepsilon_{p}$ is the effective plastic strain.

In [46], the CTOA (crack tip opening angle) criterion was adopted, with a critical value of $5^{\circ}$ and in [47] the Rankine criterion was employed.

We used two different refinements, 9400 and 37,500 particles and tested the two cracking approaches. A series of deformation plots for the fine discretization of the cracking particle 


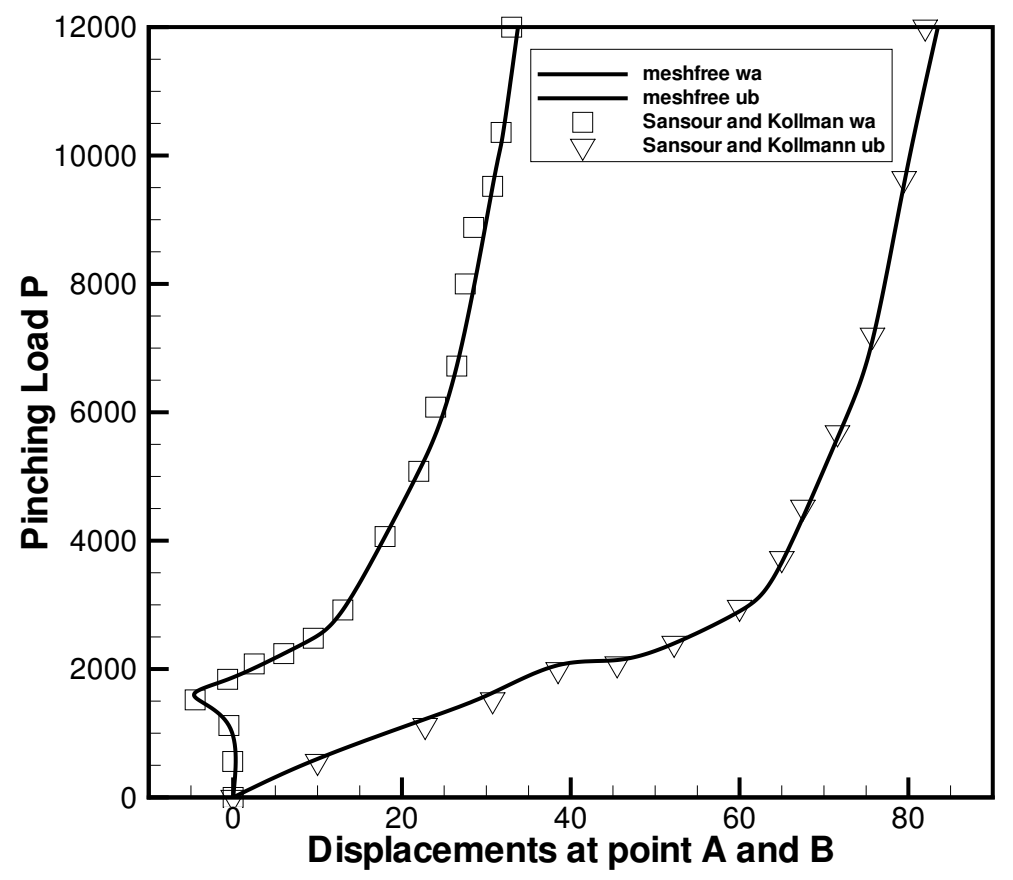

Figure 12. Load deflection curve of the elastic pinched cylinder with end diaphragms compared to the results of Sansour and Kollmann [40]

method is shown in figures 19. The results for the corresponding finer and coarser discretization, respectively, are almost identical. Also the deformation for the two different crack modeling methods is quite similar. Differences occur when the crack branches. For the cracking particle method, a smooth transition is observed and a corner between the branched cracks is separated from the cylinder. Branching occurs relatively early. For the $P U$ method, the crack branches in a much later stage, close to the tear straps, which is in better agreement with the experimental results, see also figure 20 .

The crack opening versus the pressure for the local $P U$ method is shown in figure 20 and shows very good agreement to the experiments. For the cracking particle method, the agreement is not too good since crack branching occurs too early.

6.3.3. Impacted cylinder filled with and without water A series of experiments of thin empty and water-filled containers subjected to impact loading was studied by Timm [48]. In the experiments, the container thickness $(0.6 \mathrm{~mm}-1.0 \mathrm{~mm})$ as well as the impact velocity $(300 \mathrm{~m} / \mathrm{s}-750 \mathrm{~m} / \mathrm{s})$ were varied. In all experiments, the containers were perforated by a projectile that penetrated at mid-height. The experiments revealed that for high impact velocities $(>700 \mathrm{~m} / \mathrm{s})$, the failure pattern of water-filled cylinders differs substantially from 


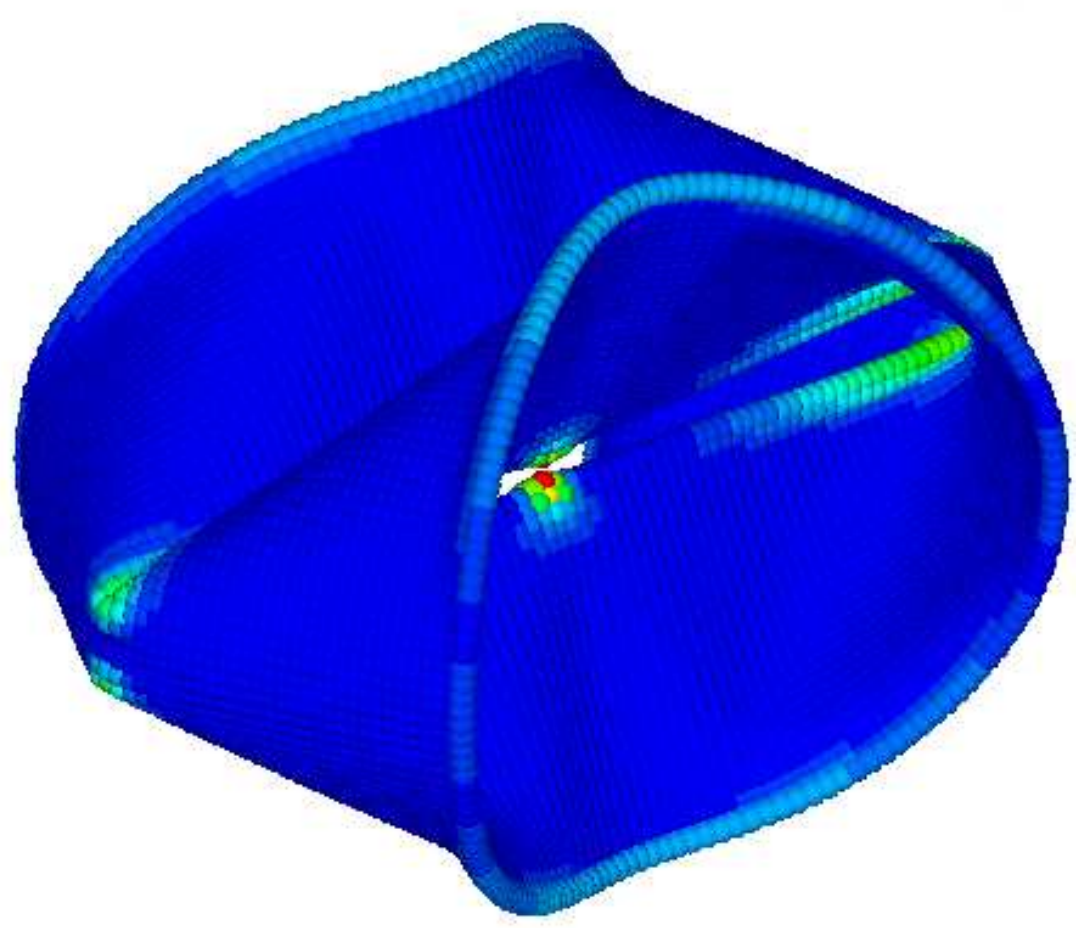

Figure 13. Deformed plastic pinched cylinder with end diaphragms

that of the empty cylinders. While a small hole was usually punched into the cylinder for low impact velocities or empty cylinders, for high impact velocities, the water filled containers crack over almost the entire length at the side where the projectile enters; (see figure 22a), for an impact velocity around $730 \mathrm{~m} / \mathrm{s}$. When the container is completely filled with water, a double triangle shaped crack opening is typical.

We consider empty and water filled containers. We used the Johnson-Cook [49] model. The material data is given by Timm [48]: $A=175 \mathrm{KN} / \mathrm{cm}^{2}, B=380 \mathrm{KN} / \mathrm{cm}^{2}, n=0.32, m=0.55$, $T_{m}=1538 C, T_{r}=25 C$ and fracture energy $G_{f}=22,000 \mathrm{~J} / \mathrm{m}^{2}$.

An exponential cohesive law described in section 5.3 is used. We study two different refinements with 29,000 and 114,000 particles for the cylinder. The deformed cylinder including the deformed projectile at $0.6 \mathrm{~ms}$ is shown in figure 21a. The principal failure mechanism is reproduced well. In figure $21 \mathrm{~b}$ the deformed water filled cylinder is shown. A severe failure at the entrance side occurs that was also observed in the experiments.

In figure 22, the final deformation of the cylinder is compared to the experiments. Figure $22 \mathrm{~d}$ shows the deformed cylinder if the failure is modelled by softening to zero stress; a length scale parameter was introduced. As can be seen, the double-triangle crack opening typical for Timm's experiments cannot be reproduced. The deformed cylinder for the cracked particle method is shown in figure $22 \mathrm{~b}$ ) and c). The results for the PU cracking method are similar. 


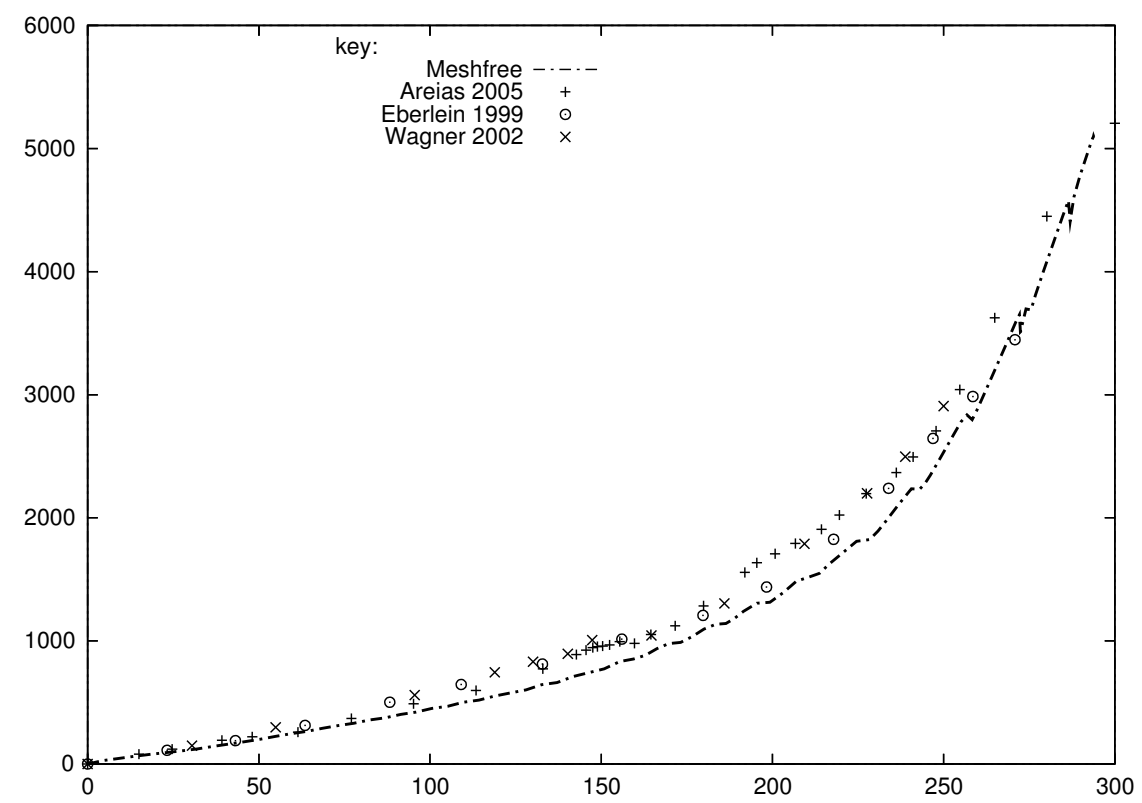

Figure 14. Load deflection curve of the plastic pinched cylinder with end diaphragms compared to the results of Areias et al. [26], Eberlein and Wriggers [43], Wagner et al. [44]

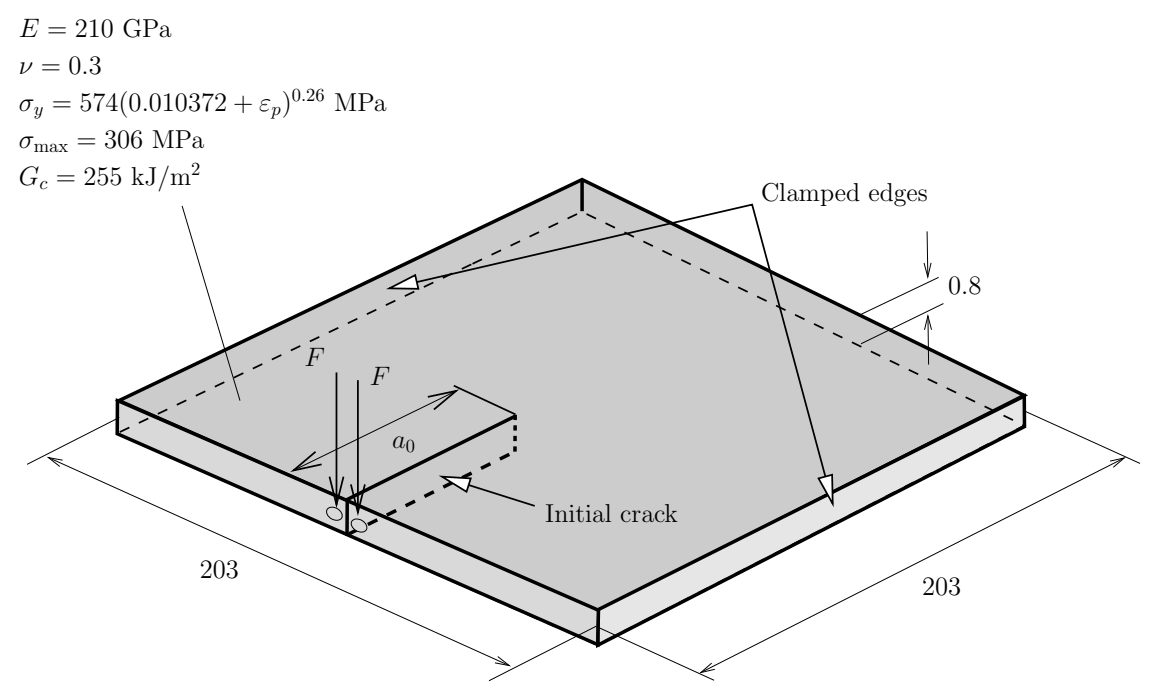

All linear dimensions are in $\mathrm{mm}$

Figure 15. Test setup of the out-of plane plate tearing problem, [45] 


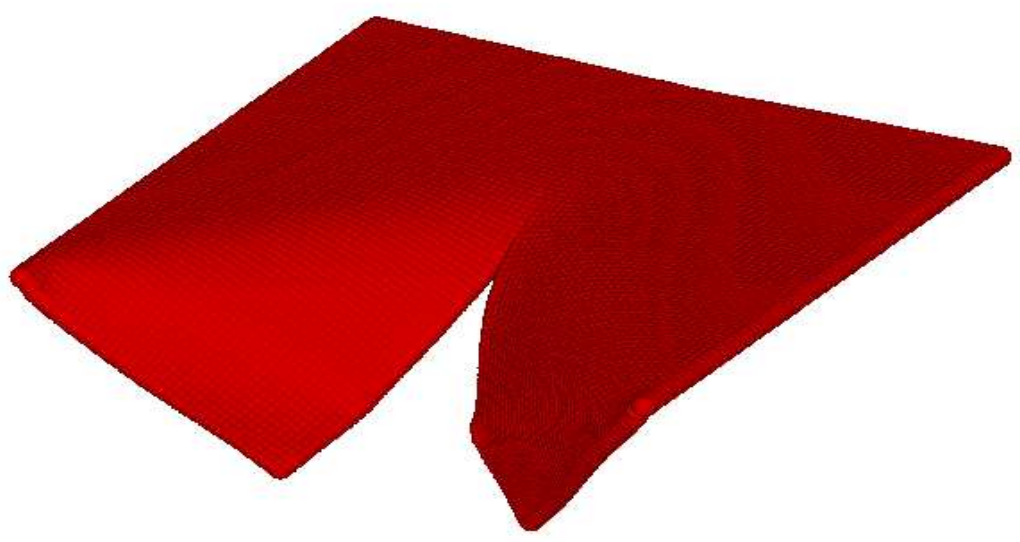

Figure 16. Deformed torn out-of plane loaded plate for a value of $a_{0}=40 \mathrm{~mm}$

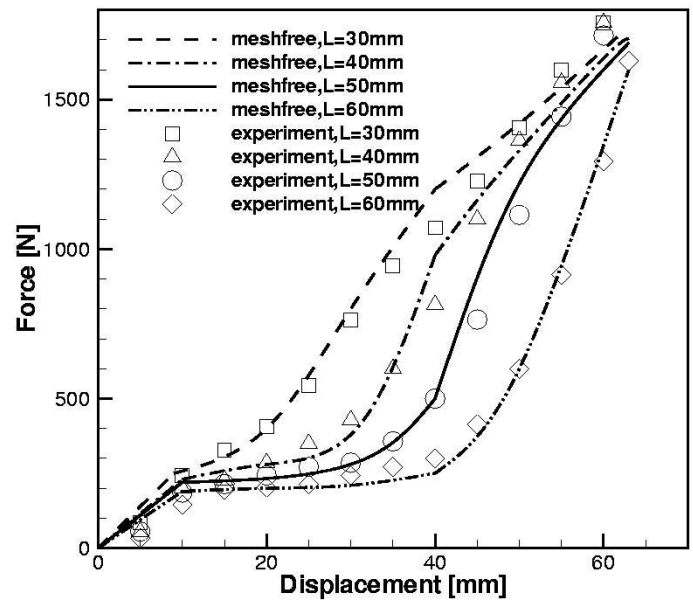

(a)

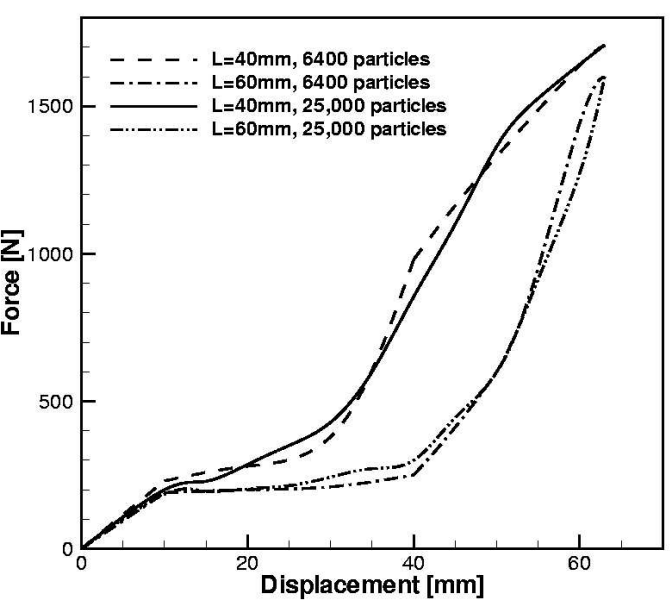

(b)

Figure 17. a) Load deflection curves for different values of $a_{0}$ for the torn out-of plane loaded plate compared to experimental data of Muscat-Fenech and Atkins [45] and b) Influence of the refinement 

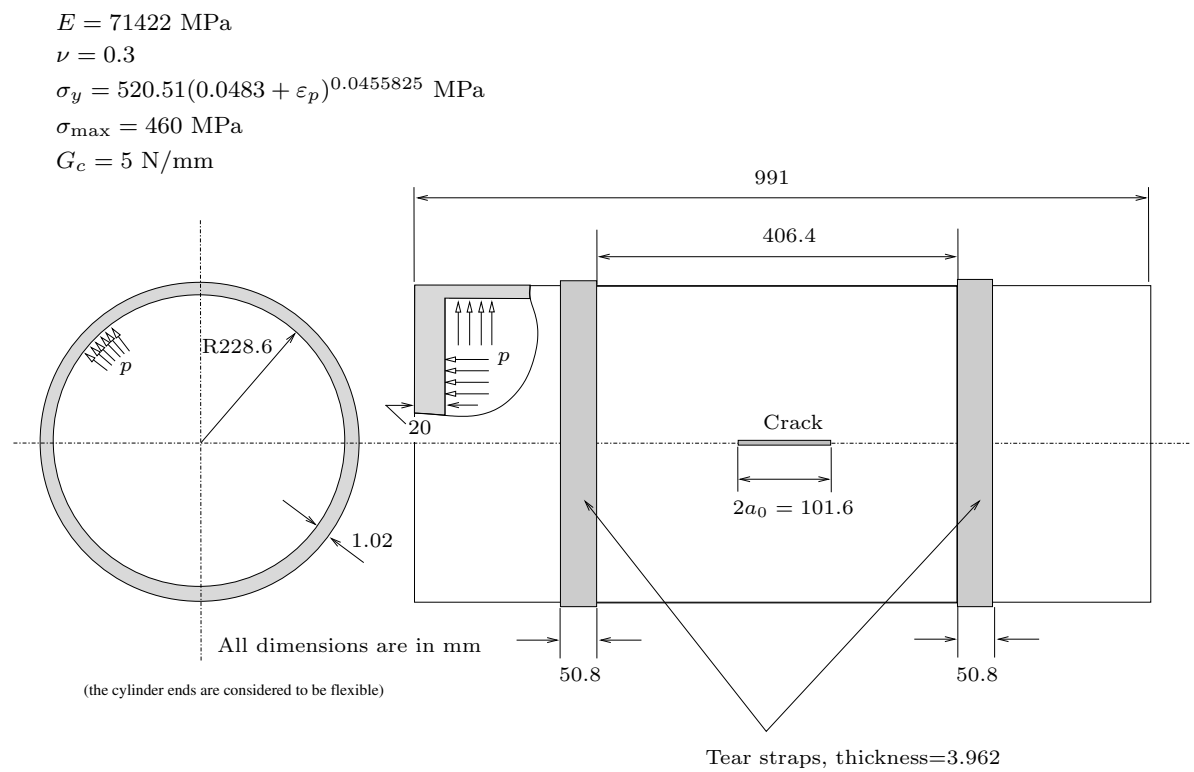

Figure 18. Relevant data for the pressurized shell problem, see also references [46, 47]

Both methods are able to reproduce the shape of the crack. Note that due to fabrication errors, the cylinder shown failed also across a weld at the top. This failure was not observed in several other experiments.

\section{CONCLUSIONS}

We have presented a meshfree thin shell formulation for finite strains and its extension to plasticity and crack modelling. Due to higher order displacement continuity of meshfree methods, when compared with finite elements, the incorporation of strong discontinuities is especially simple. We proposed two methodologies, a cracking particle method [8, 13] where the crack is introduced at the particle position and a local partition of unity $P U$ method as in Ventura et al. [11]. In the latter case, the crack is modelled as a continuous line. A methodology allowing the adoption of continuum constitutive models in the shell framework was presented, see also Areias et al. [19].

Local patches are used for the surface parametrization. The method required a parametric $C^{1}$ description of the initial shape. For the problems considered here, namely cylinders and spheres or portions thereof, this is straightforward. For more complex shapes, B-spline representations (or similar CAD techniques) would need to be used. While this was a marked disadvantage several decades ago, it is quite straightforward today, though techniques for identifying neighbors on adjacent patches would need to be developed.

We tested the method and the implementation with linear and nonlinear benchmark problems. No membrane locking was observed and the results showed good accuracy in 


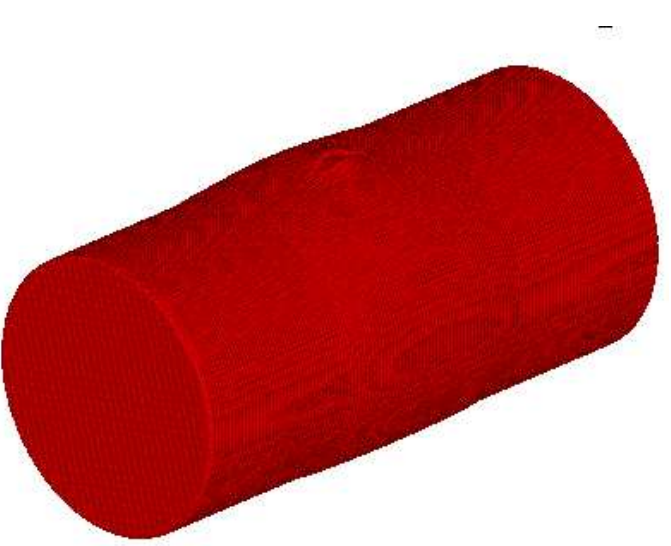

a)

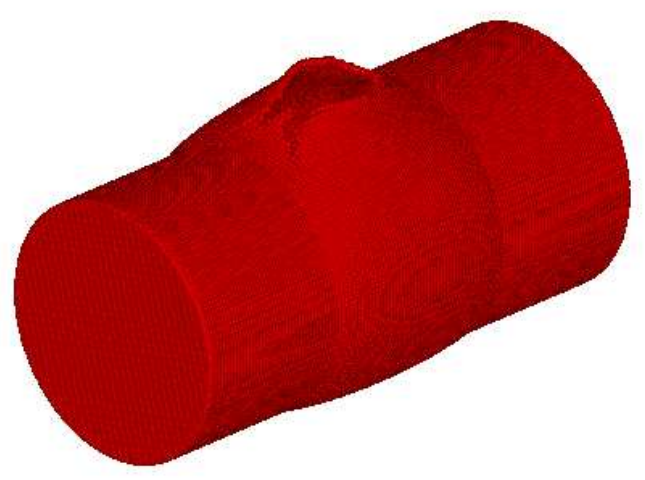

c)

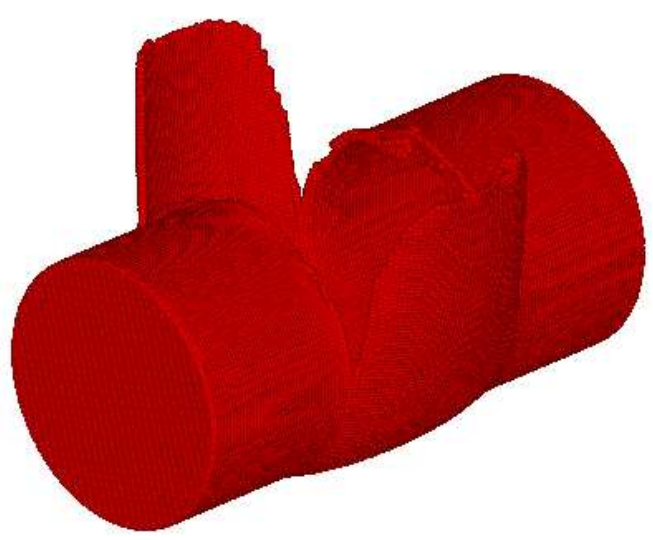

e)

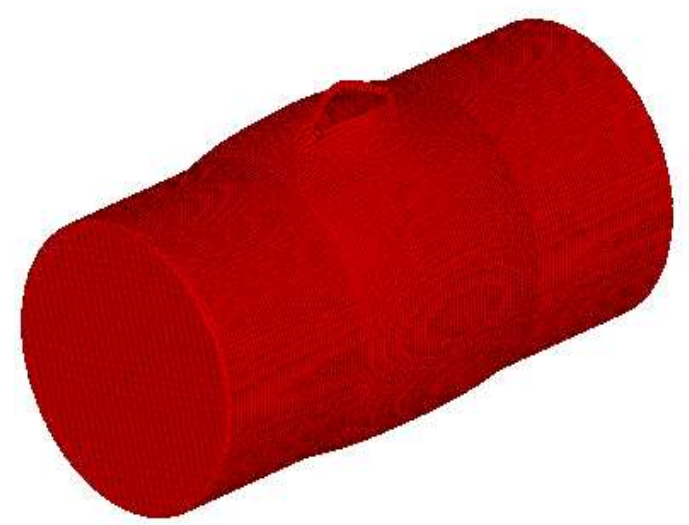

(b)

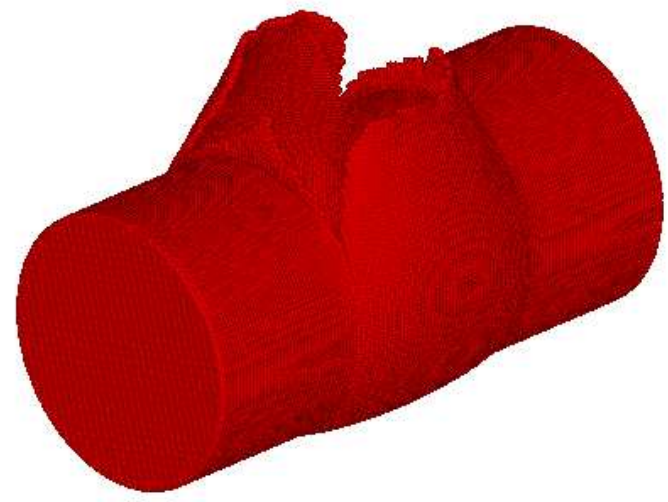

d)

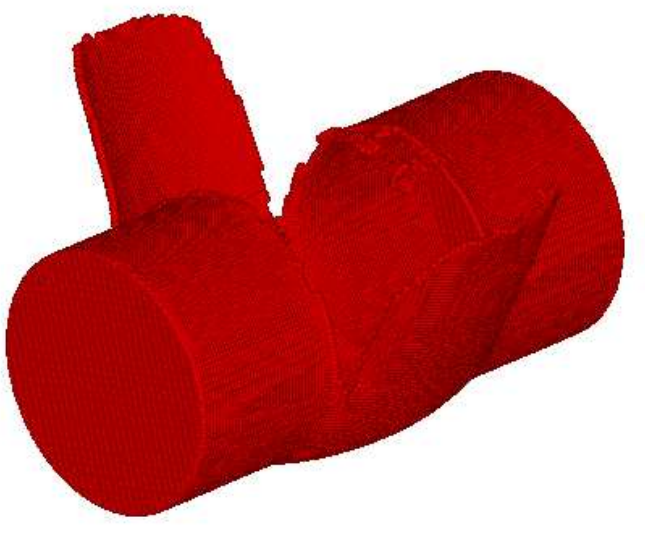

f)

Figure 19. Deformed Keesecker et al. [46] cylinder for 37,500 particles and the cracking particle method at different times 


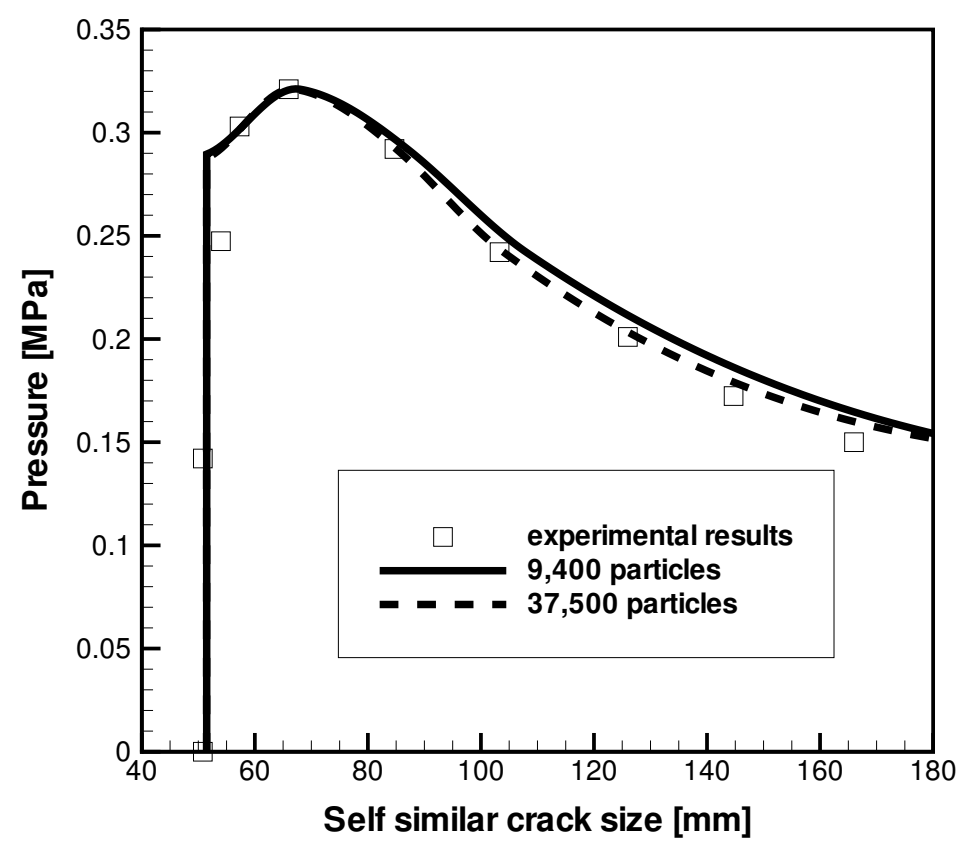

Figure 20. Self similar crack opening versus pressure for the Keesecker et al. [46] problem

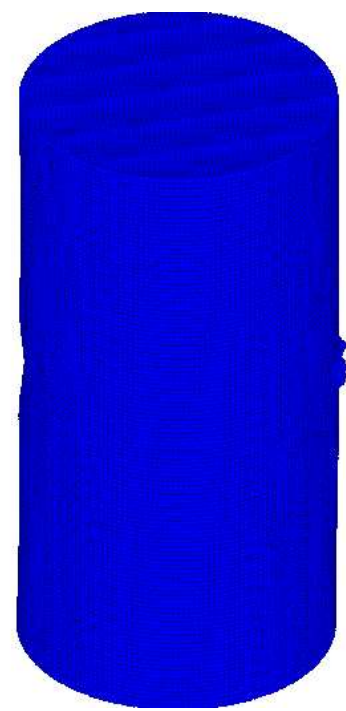

a) without water

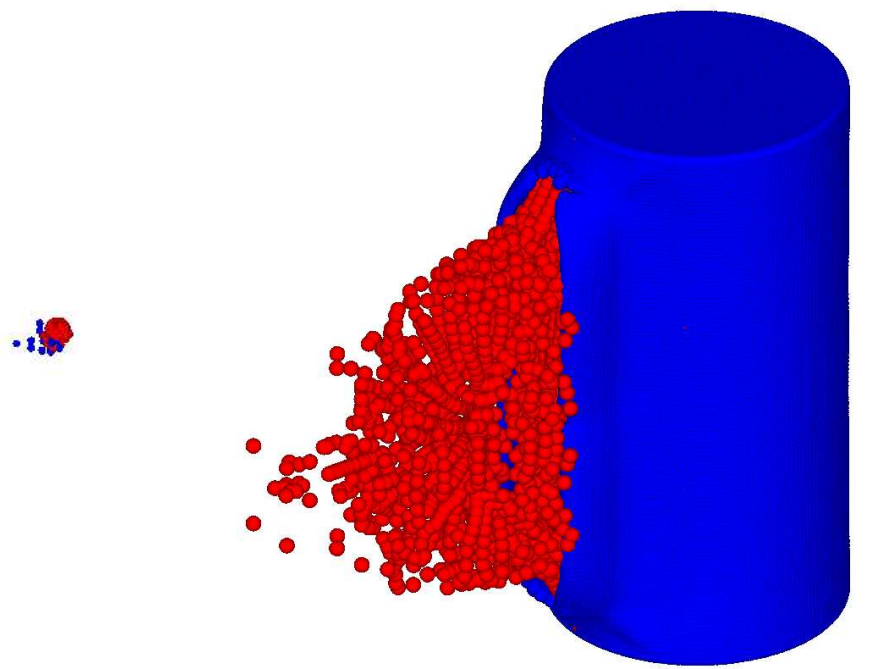

(b) with water

Figure 21. Deformation of the cylinder 


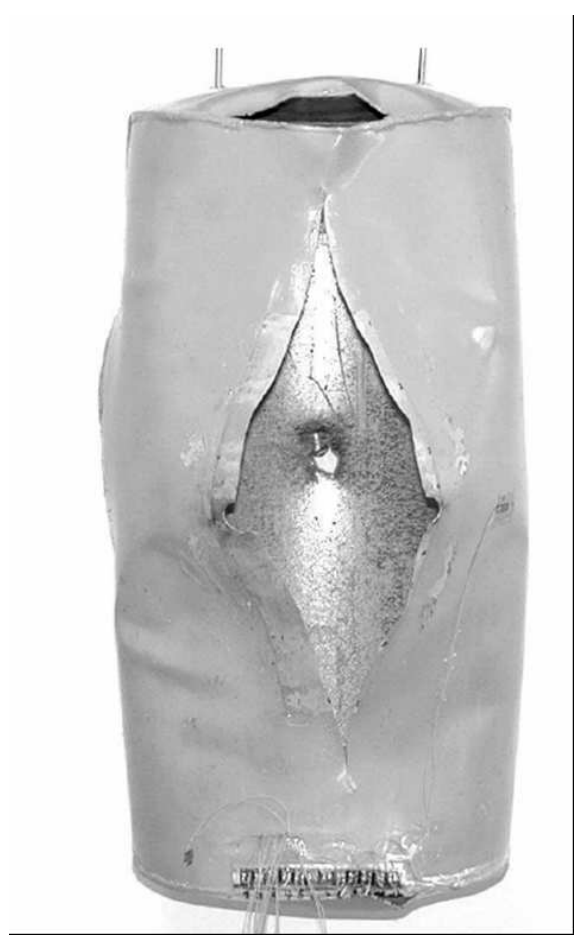

a) experiments

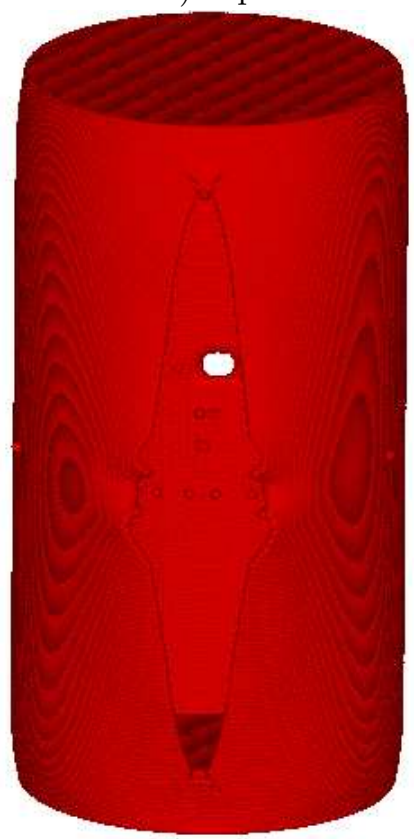

(c) 114,000 particles

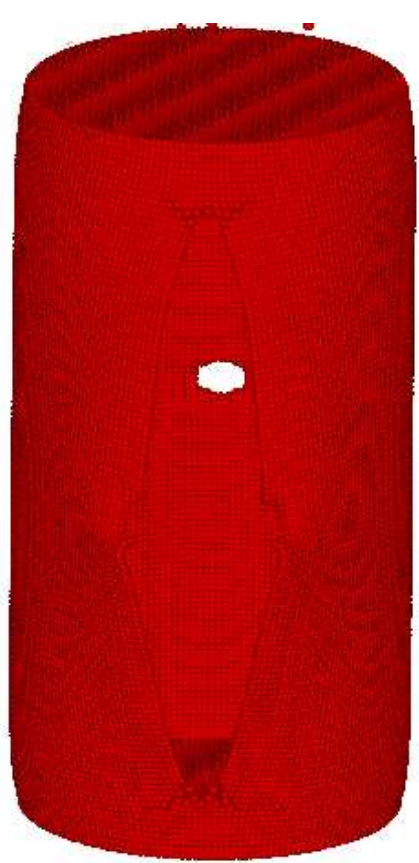

b) 29,000 particles

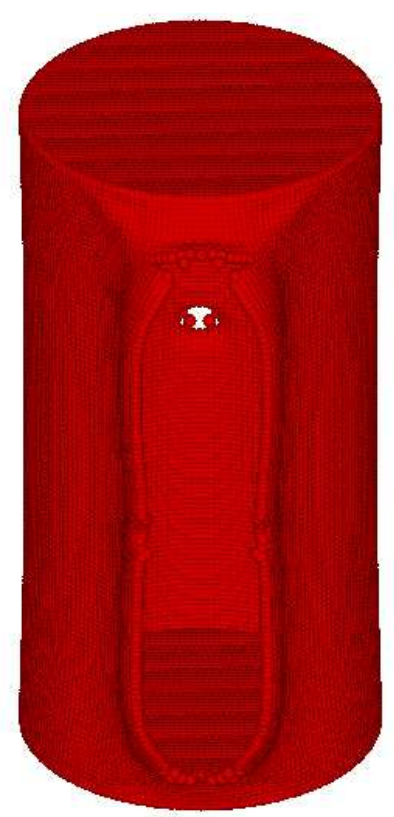

(f) 29,000 particles

Figure 22. Deformed impacted full cylinder for different methods compared to a) the experimental result);b) and c) cracking particle method, d) "smeared" crack method

Copyright (c) 2000 John Wiley \& Sons, Ltd.

Int. J. Numer. Meth. Engng 2000; 00:1-6 
comparison with other results from the literature.

The method was also applied to static and dynamic crack propagation. We found that the cracking particle method is well suited for a qualitative prediction though in some cases the quantitative agreement with epxeriments was not as good as for the PU method. This is probably due to the large domain of influence that is necessary for the higher order meshfree shape functions that are needed for the higher order approximations. An alternative would be to use Shepard functions and an extrinsic basis with additional degrees of freedom to meet quadratic or higher order completeness. This is a topic of ongoing investigations.

The $P U$ method was able to give good quantitative results as compared to experiments. This is probably due to the fact that linear crack opening can be reproduced more correctly than with the cracking particle method. However, difficulties occur for high velocity dynamic problems with multiple cracking and fragmentation since certain criteria and assumptions have to be made as to how to branch and join the cracks.

\section{ACKNOWLEDGEMENT}

The support of Office of the Naval Research under Grant N00014-03-1-0097 and the Army Research Office under Grant DAAD19-02-01-0339 is gratefully acknowledged. 
references

[1] P. Krysl and T. Belytschko. Analysis of thin shells by the element-free galerkin method. International Journal of Solids and Structures, 33(20-22):3057-3078, 1996.

[2] H. Noguchi, T. Kawashima, and T. Miyamura. Element free analysis of shell and spatial structures. International Journal for Numerical Methods in Engineering, 47(6):1215-1240, 2000 .

[3] S. Li, W. Hao, and W.K. Liu. Numerical simulations of large deformation of thin shell structures using meshfree methods. Computational Mechanics, 25(2-3):102-116, 2000.

[4] N.H. Kim, K.K. Choi, J.S. Chen, and M.E. Botkin. Meshfree analysis and design sensitivity analysis for shell structures. International Journal for Numerical Methods in Engineering, 53(9):2087-2116, 2002.

[5] O. Garcia, E.A. Fancello, C.S. de Barcellos, and C.A. Duarte. hp-clouds in Mindlin's thick plate model. International Journal for Numerical Methods in Engineering, 47(8): 1381-1400, 2000.

[6] D.D. Wang and J.S. Chen. Locking-free stabilized conforming nodal integration for meshfree Mindlin-Reissner plate formulation. Computer Methods in Applied Mechanics and Engineering, 193(12-14):1065-1083, 2004.

[7] W. Kanok-Nukulchai, W. Barry, and K. Saran-Yasoontorn. On elimination of shear locking in the element-free galkerin method. International Journal for Numerical Methods in Engineering, 52(7):705-725, 2001.

[8] T. Rabczuk and T. Belytschko. Cracking particles: A simplified meshfree method for arbitrary evolving cracks. International Journal for Numerical Methods in Engineering, 61(13):2316-2343, 2004

[9] T. Belytschko, N. Moes, S. Usui, and C. Parimi. Arbitrary discontinuities in finite elements. International Journal for Numerical Methods in Engineering, 50(4):993-1013, 2001.

[10] N. Moes, J. Dolbow, and T. Belytschko. A finite element method for crack growth without remeshing. International Journal for Numerical Methods in Engineering, 46(1):133-150, 1999.

[11] G. Ventura, J. Xu, and T. Belytschko. A vector level set method and new discontinuity approximations for crack growth by efg. International Journal for Numerical Methods in Engineering, 54(6):923-944, 2002.

[12] T. Belytschko, W. K. Liu, and B. Moran. Nonlinear Finite Elements for Continua and Structures. John Wiley and Sons, Chichester, 2000.

[13] T. Rabczuk and T. Belytschko. A three dimensional large deformation meshfree method for arbitrary evolving cracks. Computer Methods in Applied Mechanics and Engineering, accepted. 
[14] T. Rabczuk and T. Belytschko. Application of meshfree particle methods to static fracture of reinforced concrete structures. accepted in International Journal of Fracture.

[15] T. Belytschko, T. Rabczuk, E. Samaniego, and P.M.A. Areias. A simplified meshfree method for shear bands with cohesive surfaces. International Journal for Numerical Methods in Engineering, submitted.

[16] T. Belytschko, Y.Y. Lu, and L. Gu. Element-free galerkin methods. International Journal for Numerical Methods in Engineering, 37:229-256, 1994.

[17] T. Belytschko and Y.Y. Lu. Element-free galerkin methods for static and dynamic fracture. International Journal of Solids and Structures, 32:2547-2570, 1995.

[18] A. Huerta, Belytschko T., Fernandez-Mendez S., and Rabczuk T. Encyclopedia of Computational Mechanics, chapter Meshfree Methods. John Wiley and Sons, 2004.

[19] P.M.A. Areias, J.H. Song, and T. Belytschko. Analysis of fracture in thin shells by overlapping paired elements. International Journal for Numerical Methods in Engineering, 195:5343-5360, 2006.

[20] J-H Song, P.M.A. Areias, and T. Belytschko. A method for dynamic crack and shear band propagation with phantom nodes. International Journal for Numerical Methods in Engineering, 2006.

[21] J.C. Simo, D.D. Fox, and M.S. Rifai. On a stress resultant geometrically exact shell model. Part II: The linear theory; computational aspects. Computer Methods in Applied Mechanics and Engineering, 73:53-92, 1989.

[22] H. Parish. An investigation of a finite rotation four node assumed strain shell element. International Journal for Numerical Methods in Engineering, 31:127-150, 1991.

[23] M.L. Bucalem and K-J. Bathe. Higher-order MITC general shell elements. International Journal for Numerical Methods in Engineering, 36:3729-3754, 1993.

[24] R. Hauptmann and K. Schweizerhof. A systematic development of solid-shell element formulations for linear and non-linear analyzes employing only displacement degrees of freedom. International Journal for Numerical Methods in Engineering, 42:49-69, 1998.

[25] T. Belytschko, H. Stolarski, W.K. Liu, N. Carpenter, and J.S.J. Ong. Stress projection for membrane and shear locking in shell finite-elements. Computer Methods in Applied Mechanics and Engineering, 51:221-258, 1985.

[26] P.M.A. Areias, J.-H. Song, and T. Belytschko. A finite-strain quadrilateral shell element based on discrete Kirchhoff-Love constraints. International Journal for Numerical Methods in Engineering, 64:1166-1206, 2005.

[27] K.Y. Sze, S.H. Lo, and L.Q. Yao. Hybrid-stress solid elements for shell structures based upon a modified variational functional. International Journal for Numerical Methods in Engineering, 53:2617-2642, 2002. 
[28] P.M.A. Areias, J.M.A. César de Sá, and Conceio António. A gradient model for finite strain elastoplasticity coupled with damage. Finite Elements in Analysis and Design, 39: 1191-1235, 2003.

[29] M.L. Bucalem and K-J. Bathe. Higher-order MITC general shell elements. International Journal for Numerical Methods in Engineering, 36:3729-3754, 1993.

[30] R. Hauptmann and K. Schweizerhof. A systematic development of solid-shell element formulations for linear and non-linear analyzes employing only displacement degrees of freedom. International Journal for Numerical Methods in Engineering, 42:49-69, 1998.

[31] S.L. Weissmann. High-accuracy low-order three-dimensional brick element. International Journal for Numerical Methods in Engineering, 39:2337-2361, 1996.

[32] M.A. Crisfield and X. Peng. Instabilities induced by coarse meshes for a nonlinear shell problem. Engineering Computations, 13:110-114, 1996.

[33] P.M.A. Areias, J.H. Song, and T. Belytschko. A simple finite-strain quadrilateral shell element part i: Elasticity. International Journal for Numerical Methods in Engineering, in press, 2005.

[34] A. Ibrahimbegović, B. Brank, and P. Courtois. Stress resultant geometrically exact form of classical shell model and vector-like parameterization of constrained finite rotations. International Journal for Numerical Methods in Engineering, 52:1235-1252, 2001.

[35] A. Eriksson and C. Pacoste. Element formulation and numerical techniques for stability problems in shells. Computer Methods in Applied Mechanics and Engineering, 191:3775$3810,2002$.

[36] X. Peng and M.A. Crisfield. A consistent co-rotational formulation for shells using the constant stress/constant moment triangle. International Journal for Numerical Methods in Engineering, 35:1829-1847, 1992.

[37] C. Sansour and F.G. Kollmann. Large viscoplastic deformations of shells. Theory and finite element formulation. Computational Mechanics, 21:512-525, 1998.

[38] X. Peng and M.A. Crisfield. A consistent co-rotational formulation for shells using the constant stress/constant moment triangle. International Journal for Numerical Methods in Engineering, 35:1829-1847, 1992.

[39] A. Masud, C.L. Tham, and W.K. Liu. A stabilized 3D co-rotational formulation for geometrically nonlinear analysis of multi-layered composite shells. Computational Mechanics, 26:1-12, 2000.

[40] C. Sansour and F.G. Kollmann. Families of 4-node and 9-node finite elements for a finite deformation shell theory. an assessment of hybrid stress, hybrid strain and enhanced strain elements. Computational Mechanics, 24:435-447, 2000.

[41] E.M.B. Campello, P.M. Pimenta, and P. Wriggers. A triangular finite shell element based on a fully nonlinear shell formulation. Computational Mechanics, 31:505-518, 2003. 
[42] P.M.A. Areias. Finite element technology, damage modeling, contact constraints and fracture analysis. Doutoramento, FEUP - Faculdade de Engenharia da Universidade do Porto, Rua Dr. Roberto Frias s/n 4200-465 Porto, Portugal, 2003. www.fe.up.pt.

[43] R. Eberlein and P. Wriggers. Finite element concepts for finite elastoplastic strains and isotropic stress response in shells: Theoretical and computational analysis. Computer Methods in Applied Mechanics and Engineering, 171:243-279, 1999.

[44] W. Wagner, S. Klinkel, and F. Gruttmann. Elastic and plastic analysis of thin-walled structures using improved hexahedral elements. Computers and Structures, 80:857-869, 2002.

[45] C.M. Muscat-Fenech and A.G. Atkins. Out-of-plane stretching and tearing fracture in ductile sheet-materials. International Journal of Fracture, 84:297-306, 1997.

[46] A.L. Keesecker, C.G. Davila, E.R. Johnson, and J.H. Starnes Jr. Crack path bifurcation at a tear strap in a pressurized shell. Computers and Structures, 81:1633-1642, 2003.

[47] P.M.A. Areias and T. Belytschko. Analysis of three-dimensional crack initiation and propagation using the extended finite element method. International Journal for Numerical Methods in Engineering, 63:760-788, 2005.

[48] T. Timm. Beschuss von fluessigkeitsgefuellten Behaeltern. PhD thesis, University of Karlsruhe, Institut fuer Massivbau und Baustofftechnologie, 2002.

[49] G.R. Johnson and W.H. Cook. A constitutive model and data for metals subjected to large strains, high strain rates, and high temperatures. In Proc. 7th International Symp. on Ballistics, 1983. 